\title{
Quality-by-design in pharmaceutical development: From current perspectives to practical applications
}

BÉLA KOVÁCS ${ }^{1}$

ORSOLYA PÉTERFI ${ }^{2}$

BOGLÁRKA KOVÁCS-DEÁK²

ISTVÁN SZÉKELY-SZENTMIKLÓSI ${ }^{2}$

IBOLYA FÜLÖP3,*

LÁSZLÓ-ISTVÁN BÁBA ${ }^{3}$

FRANCISC BODA ${ }^{1}$

${ }^{1}$ Department F1, Faculty of Pharmacy George Emil Palade University of Medicine Pharmacy, Science, and Technology of Târgu Mureș, 540142 Târgu Mures, Romania

${ }^{2}$ Faculty of Pharmacy, George Emil Palade University of Medicine, Pharmacy Science, and Technology of Târgu Mureș 540142 Târgu Mures, Romania

${ }^{3}$ Department F2, Faculty of Pharmacy George Emil Palade University of Medicine Pharmacy, Science, and Technology of Târgu, 540142 Târgu Mures, Romania

\section{Accepted November 23, 2020}

Published online December 23, 2020

\begin{abstract}
Current pharmaceutical research directions tend to follow a systematic approach in the field of applied research and development. The concept of quality-by-design $(\mathrm{QbD})$ has been the focus of the current progress of pharmaceutical sciences. It is based on, but not limited, to risk assessment, design of experiments and other computational methods and process analytical technology. These tools offer a well-organized methodology, both to identify and analyse the hazards that should be handled as critical, and are therefore applicable in the control strategy. Once implemented, the QbD approach will augment the comprehension of experts concerning the developed analytical technique or manufacturing process. The main activities are oriented towards the identification of the quality target product profiles, along with the critical quality attributes, the risk management of these and their analysis through in silico aided methods. This review aims to offer an overview of the current standpoints and general applications of $\mathrm{QbD}$ methods in pharmaceutical development.
\end{abstract}

Keywords: quality-by-design, pharmaceutical development, risk assessment, software-aided development

\section{INTRODUCTION}

The traditional approach for assuring and maintaining product quality was an empirical method based on trial and error, which grounded on a ceteris paribus concept known as the OFAT-method (one-factor-at-a-time). This approach carried the risk to incorporate the possibility of the occurrence of unexpected, out-of-specification results not only during process optimization, but also at the validation stage, which derived from insufficient product and process understanding. Although process validation is the golden standard in pharmaceutical development, presently in order to assure product quality and to gain background knowledge and thorough process understanding, the International Conference on Harmonization of Technical Requirements for Registration of Pharmaceuticals for Human Use $(\mathrm{ICH})$ recommends the use of quality-by-design $(\mathrm{QbD})$ concept (1-3). According to the

\footnotetext{
*Correspondence; e-mail: ibolya.fulop@umfst.ro
} 
ICH Q8 guideline, QbD is defined as a systematic approach to product development; more precisely, it centres on designing manufacturing processes and product formulations from the earliest possible stage of development. Implementing QbD in the research and development of pharmaceutical products requires a predefined objective and a deeper understanding of formulation and manufacturing process variables. Identifying the causes of quality issues through the understanding of the aforementioned variables ensures the implementation of a control strategy, and the development of a pharmaceutical product with pre- and well-defined quality standards which aims to match patients' desideratum, and thus assuring the proper compliance.

The aim of this work is to summarize the current viewpoints regarding the position of $\mathrm{QbD}$ in pharmaceutical development and its application in both analytical and technological (manufacturing) context. Thus, this work represents a useful starting point for specialists in the pharmaceutical industry to become familiarized with QbD terminology, offering examples for the use of these methods in various contexts, as well as indicating other comprehensive reviews that expound specific aspects of this concept.

\section{CURRENT REGULATORY DEMANDS AND TRENDS IN PHARMACEUTICAL DEVELOPMENT REGARDING QUALITY-BY-DESIGN}

Requirements for product quality are described in the tripartite guidelines of ICH, i.e., Q8 (pharmaceutical development), Q9 (quality risk assessment) and Q10 (pharmaceutical quality system). The ICH Q8 guideline establishes the elements of pharmaceutical development, namely, the quality target product profile (QTPP), critical quality attributes (CQAs), critical process parameters (CPPs), risk assessment (RA), design space, control strategy and product life-cycle management. The ICH Q9 guideline discusses the general principles of risk management and the use of risk analysis tools, whereas the ICH Q10 guideline provides guidance for science- and risk-based regulatory approaches, in addition to quality management (3-5).

In the past decade, further guidelines have been elaborated by $\mathrm{ICH}$, aiming to complement or clarify different concepts, strategies or regulatory demands already implemented in previously published guides (ICH Q8-Q10). In this view, ICH Q11 supplements the information mainly, but not exclusively, described in the tripartite guideline of ICH Q8, Q9 and Q10, concerning the identification of quality target product profiles (QTPPs) and critical quality attributes (CQAs), development of experimental design space and appropriate control strategies for quality assurance throughout product life-cycle (6). In addition, the implemented ICH Q12 extends the use of QbD in pharmaceutical life-cycle management in the commercial phase, in terms of post-approval change management in CMCs (chemistry, manufacturing and controls) (7).

QTPP is the summary of the product's quality characteristics in regard to safety and efficacy, taking into account the route of administration, dosage form, delivery system, dosage strength, drug quality criteria (sterility, purity and stability) and others.

CQAs are physical, chemical, biological, or microbiological properties associated with the drug substance, excipients, intermediates or drug product that should be within an appropriate limit, range or distribution. The identification of the product and intermediate CQAs is followed by the determination of material attributes and process parameters that 


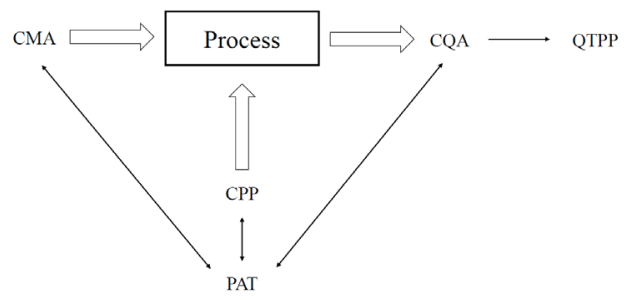

Scheme 1

may affect CQAs. Process parameter variables influencing CQAs are defined as critical process parameters (CPPs) that should be monitored or controlled to ensure quality. Critical material attributes (CMAs) are not defined by the ICH guidelines; however, they are still used as physical, chemical, biological, or microbiological properties of the input materials that should be within an appropriate limit.

Process analytical technology (PAT) is a system for monitoring the aforementioned attributes (CQA, CMA and CPP) while increasing the efficiency of detecting failures compared to end-product testing, therefore minimizing the volume of rejected products (8).

Recently two concept papers have been elaborated by the ICH, namely, ICH Q13 focusing on continuous manufacturing and ICH Q14 dealing with the analytical QbD approach. ICH Q14 comes into the revision of the ICH Q2 guideline and aims to compensate for the lack of analytical development viewpoints in the $\mathrm{ICH}$ terminology, and to cover new and innovative areas of analytical method development (e.g., near-infrared or Raman spectroscopy) $(9,10)$. The connection between the attributes presented above, namely, relationships between input CMA, CPP, output CQA and system monitoring PAT are given in Scheme 1.

Despite QbD not yet being mandatory for product development and manufacturing, this approach and/or some of its elements have been adopted in the pharmaceutical industry, with RA being one the most frequently used tool next to experimental designs (11). In a systematic review presented by Grangeia et al. (12) RA as a $\mathrm{QbD}$ tool was performed in almost $50 \%$ of cases, whereas screening, or optimizing the design of experiments (DoEs) were conducted in almost $75 \%$ according to the presented literature survey, making the latter one the most frequently used $\mathrm{QbD}$ tool in pharmaceutical development. In a timeline of QbD implementation RA, followed by DoE, assisted or not by other in silico techniques (e.g., multivariate data analysis), generally contributes to the definition of a design space where operable control strategies (e.g., PAT tools) can be implemented in order to assure product performance.

\section{RISK ASSESSMENT METHODS FOR CONTROL STRATEGY OF PHARMACEUTICAL PROCESSES}

As mentioned before, quality by design based on RA is widely used in pharmaceutical R\&D. In the past decades, various RA methods have been proposed in order to evaluate, identify and eventually control hazards that might have a critical impact on general processes and/or product quality. The ICH Q9 guideline stipulates a typical process for risk 
management, generally including five important steps during the implementation of a RA, as follows (4):

(i) risk initiation - in this step the definition of the potential hazard on the process is formed and an established core team will set up a timeline, in order to evaluate, control and communicate the outcome of the risk management process;

(ii) RA - including the identification, analysis and evaluation of risks, using different RA methods (preliminary hazard analysis - PHA, fishbone/Ishikawa chart, failure mode and effects analysis - FMEA, failure mode and effects critical analysis - FMECA, fault tree analysis - FTA, hazard analysis and critical control points - HACCP, risk ranking and filtering matrices);

(iii) risk control - comprising of risk reduction, generally through several preventive measurements, or risk acceptance, when hazard mitigation through the implemented actions is not satisfactory;

(iv) risk communication - disclosure of the outputs obtained after risk analysis and control;

(v) risk review - this step represents the re-evaluation of the risk management, as new and much vaster knowledge and experience are obtained about the process.

Amongst the various RA methods, PHA, fishbone charts, FMECA and FTA are the most widely used in pharmaceutical development-related hazard control.

Preliminary hazard analysis (PHA). - The PHA is a qualitative RA tool, which is often used to initiate the risk analysis. Its main advantage lies in the rapid overview of the major risks in the early lifecycle of the development, although in a non-systematic way. Risk evaluation through PHA requires general brainstorming techniques, as it is based on the previous experiences of the team members $(13,14)$. Therefore, PHA is not sufficient when solely used, it must be followed by comprehensive analysis, e.g., hazard and operability study (HAZOP), or FMECA (15). The tool offers a tabular overview, containing, but not limited to, information about the hazard itself, a list of identified accidents, accident risk classification, description of system functions and human error contribution to accidents leading to hazardous events (15).

Cause and effect diagram. - Cause and effect diagram (Ishikawa diagram, fishbone chart) was developed by Karou Ishikawa in the late 1960s, being one of the most frequently used RA techniques nowadays. The chart is composed of two major parts. The "head of the fish" represents the CQAs, which have to be controlled, whilst the "body with ribs" contains the underlying causes that, left uncontrolled, might lead to the defined failure (16). The main branches, or primary causes, are commonly based on six basic categories, known as the six Ms (materials, man, machine, milieu, measurements and method) (17). The technique offers a general but systematic overview of the potential hazards (Fig. 1) and it is frequently associated with other RA techniques, oftentimes FMECA.

Failure mode and effects critical analysis (FMECA). - FMECA is one of the most important RA tools, used to identify the hazards and threats that can affect the defined aims and objectives of a process (18). The method uses the analysis and categorization of the potential risks based on their severity, occurrence and detectability on an arbitrary scale defined by the working group employed in the evaluation tasks. Afterwards, a risk prioritizing 


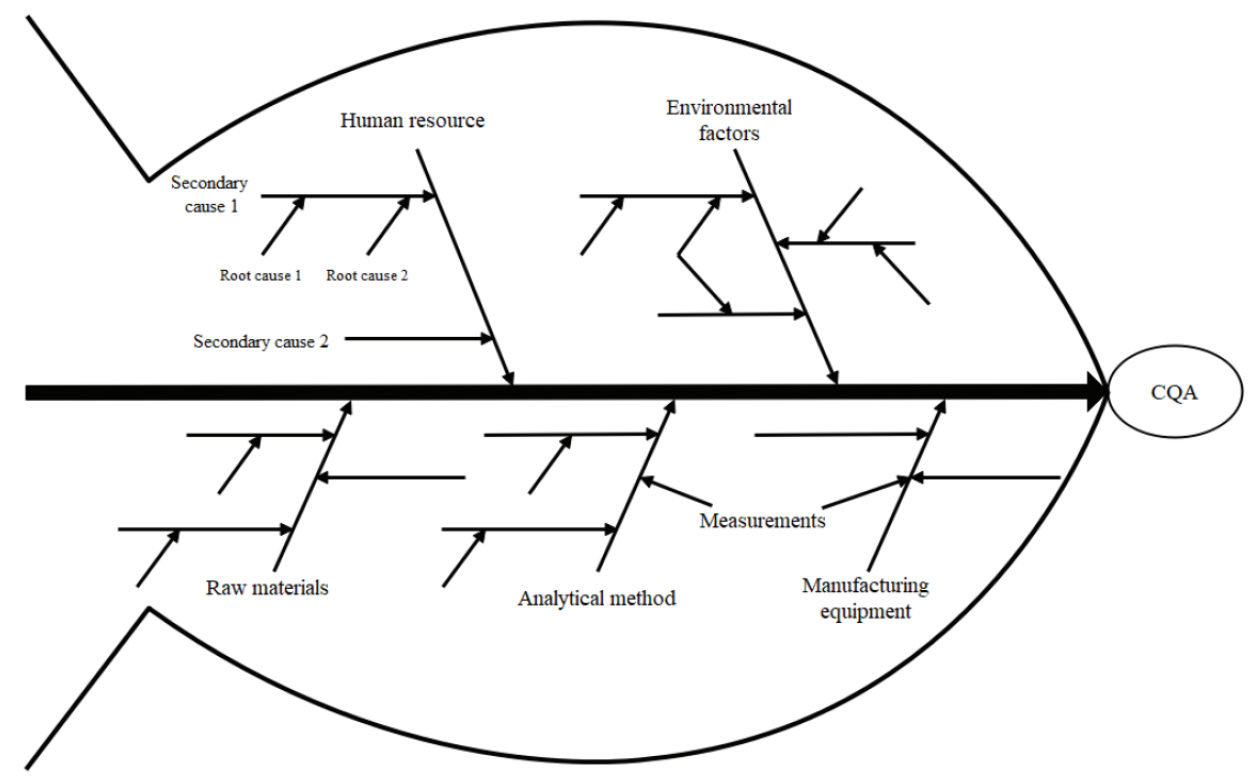

Fig. 1. A general sketch representing hazard analysis applying the Ishikawa diagram. A putative or empirically observed defect might have several primary causes, which can be systematically analysed. Conclusively, the root causes can be identified and the working group can implement proper control strategies.

number is calculated as a product of the three characteristics of the hazard. Preventive and corrective measurements will be formulated and implemented only for those process parameters that are considered as candidates for carrying a risk. In this view, FMECA is a valuable tool for risk management and is recommended as a complementary activity for method validation in both analytical $(19,20)$ and technological processes $(21)$.

Fault tree analysis (FTA). - FTA, in contrast to FMECA, is a top-down RA method, which seeks to uncover the root cause of a risk. FTA is frequently combined with FMECA in the risk management approach to analyse hazards (22). This diagrammatic analysis applies Boolean logic gates (e.g., and, or, inhibit) and transfer symbols in order to establish relationships between lower-level events, such as basic, conditioning, intermediate and external events (23).

Application of RA in pharmaceutical development. - Among the existing RA methods Ishikawa chart and FMECA analysis are the most preferred methods in pharmaceutical development and are generally used in connection with each other (12). In the current research trends, RA has been extensively used in both pharmaceutical manufacturing and analytical method development.

A pertinent example for RA-based development as an integrated part of the $\mathrm{QbD}$ was elaborated by Iurian et al. (24) for a prolonged release delivery system containing paliperidone as active substance. Controllable CQAs (mass uniformity, crushing strength and in 
vitro release kinetics) for product performance were defined using a fishbone diagram based on the defined QTPPs established by preliminary studies. The CPPs underlying the identified CQAs were further analysed by FMEA. In a work presented by Mishra et al. (25) RA based on Ishikawa diagram and PHA was used in order to elucidate factors that influence the defined QTPPs in the case of carbamazepine containing orally disintegrating tablets. Zhang et al. (26) applied the Ishikawa diagram as an appropriate quality RA tool for the identification of CQAs in the case of a stability-indicating RP-HPLC method for a forced degradation study of cloxacillin. In their study, five factors $(\mathrm{pH}$ of the buffer solution, solvent type, solvent content, concentration of the buffering salt and column temperature) have been identified, which were afterwards screened for their significant effect using experimental designs. After an initial screening, with the help of Pareto charts, the factors were ranked based on their significance on method performance and were further optimized with the help of DoE, whilst the other two statistically insignificant factors were kept constant further on. An interesting approach to analyse and investigate hazards in the case of HPLC method development was presented by Reid et al. (27). The technique successfully applied both an Excel-based analysis and a software-aided approach in order to identify and categorize risks that influence a chromatographic system.

The outcome of risk analysis is generally the definition of the CQAs, which need to be analysed and controlled in order to gain in-depth knowledge and to assure proper robustness of the developed methods and manufacturing processes. For this purpose, software-assisted tools have obtained increased attention and have been extensively used. Proper risk analysis of influencing factors underlying CQAs, generally performed by a multidisciplinary team, is the fundamental prerequisite for the establishment of a subsequent DoE screening and optimization required in both analytical and technological fields.

\section{QUALITY-BY-DESIGN IN THE DEVELOPMENT OF ANALYTICAL METHODS AND PHARMACEUTICAL MANUFACTURING}

Method development strategy - a general workflow of AQbD implementation. - The concept of $\mathrm{QbD}$, widely used in the pharmaceutical industry, can be applied in development of analytical methods and validation, known as analytical quality by design (AQbD). It was introduced by the US Food and Drug Administration and reported in the ICH Q8(R2) guideline $(3,19)$, although these documents elaborated by major regulatory bodies have been preceded by pioneering studies in this field of research (28). There are many analytical methods developed using the AQbD approach, such as enantioseparation and purity determination by capillary zone electrophoresis $(29,30)$, Karl Fischer titration for determination of water content (31), spectroscopy for quantitative colour measurement (32), etc. Analytical methods that are commonly used for quality control (assay, stability, content uniformity and impurity profile) are mainly based on chromatographic techniques (HPLC, UHPLC, LC-MS, SFC, GC), thus considering the empirical relevance of these methods, in this section we propose to review the relevant information regarding the application of $\mathrm{AQbD}$ in chromatographic methods.

(i) Definition of the analytical target profiles (ATPS). - The key component and the first step of the AQbD is to determine the analytical target profile (ATP) (33), the aim of the devel- 
oped analytical method (34) without identifying the method development steps and the method itself. An up-to-date definition of ATP was formulated by Jackson et al. (35) stipulating that "ATP, in a similar fashion to the quality target product profile (QTPP), prospectively summarizes the requirements associated with a measurement on a quality attribute which needs to be met by an analytical procedure and is used to define and assess the fitness of an analytical procedure in the development phase and during all changes across the analytical lifecycle".

For an adequate ATP definition, it is important to know the matrix, the physicochemical properties of the main analyte/analytes ( $\mathrm{pK}_{\mathrm{a}}$, solubility and stability), and so on. For example, in the case of azilsartan, medoxomil and chlorthalidone hydrochloride containing tablets the ATP is the determination of these active ingredients in the presence of their degradation products, providing suitable specificity due to structural similarities (36).

Along with the specification of the scope of the method (e.g., naming the main analyte), the ATP can include the definition of some performance criteria of the method, performance characteristics of CMAs and operational factors (analysis time, specificity, relative retention time, linearity range, etc.) $(33,37,38)$. The ATP should be valid not only across the method development and validation phase but also during the method transfer, therefore, precision and accuracy of the method are frequently included as ATP criteria (39).

(ii) Definition of CMAs and CMPs and performing RA. - Critical method parameters, $\mathrm{CMPs}$ [termed as critical process parameters $(\mathrm{CPP})$ in $\mathrm{QbD}$ ] are defined as variables that affect the resulting method, which are evaluated by the critical method attributes (CMAs) [used as critical quality attributes (CQA) in $\mathrm{QbD}$ ].

In the case of an HPLC/UHPLC method, these CMAs can be peak symmetry, tailing factor, peak width, analysis time, the resolution between two peaks, theoretical plates, signal-to-noise ratio, etc. (40-43). In the case of a complex matrix (e.g., plant extract, biological matrix) the definition of CMA can be difficult (if in any condition the CMA varies between the preferred intervals, it cannot be considered a CMA) (44).

The CMPs are determined based on preliminary experiments and literature data. The most common parameters, which serve as CMPs, are column temperature, mobile phase flow rate, organic modifier (acetonitrile, methanol) percentage, gradient time, injection volume, mobile phase $\mathrm{pH}$. If an MS detector is used, there are other CMAs influencing parameters (spray voltage, sheath gas and auxiliary gas pressure) in addition to the abovementioned ones. The CMPs of GC methods can be the oven temperature and program, and carrier gas velocity (44-46).

Risk factor identification through prior knowledge, risk analysis through Ishikawa diagram, along with risk evaluation through FMECA, identify and prioritize the main factors that could influence CMAs and consequently the ATP. Risk factors can be classified as high-risk factors, which should be eliminated during the method development process, noise factors, which are recommended to be tested by robustness studies, and experimental factors, required to be studied by ruggedness test $(33,47)$. Most frequently, Ishikawa diagrams are used to help in the identification of risk factors in AQbD including the following: environment, materials, method, human factor, and machine (39).

(iii) Use of experimental designs (DoE) for method development. - DoE is a systematic approach to determine the relationship between input and output variables through 
mathematical models. The number of experiments can be reduced by systematically and purposefully varying experimental parameters to maximize the available information and identify the effects of process and product parameters on response variables. In addition, DoE is used to determine CPPs, their interactions, and identify the optimum settings of these parameters in order to maintain CQAs $(48,49)$. The NIST (National Institute of Standards and Technology) handbook identifies the following steps in the process of a DoE (50):

- determination of the objectives, which implies identifying the QTPP;

- selection of process variables, both inputs and outputs (CPPs, CQAs);

- selection of experimental design;

- execution of the experiment;

- verification of data consistency with experimental assumptions;

- analysis and evaluation of results;

- interpretation and use of the results.

There are many DoE tools, assisted by several software programs, mainly based on linear solvent strength theory (51). The most frequently used methods in practice are listed in Table I. The screening designs are applied for the selection of CMPs and CMAs and are followed by DoE experiments used for method optimization. Application of DoE is part of the $\mathrm{AQbD}$ approach, but not the only step; however, there are papers where the two terms are misinterpreted.

A recent study presented by Sylvester et al. (52) follows a step-by-step AQbD development and validation of an HPLC method for the separation of curcumins and doxorubicin from a long-circulating liposome formulation using a D-optimal experimental design. Using a gradient HPLC method the critical step in method development and thus in DoE was the optimization of the gradient elution program defining the time span for the two solvents at a given percentage, in the initial isocratic hold and purging time. In addition to the duration of the linear-gradient, the initial and final percentage of the two components of the mobile phase could also represent CMPs (44). In all cases, DoE experiments are for HPLC method fine-tuning, previously reported methods or preliminary experiments being the starting point. Numerous reverse phase HPLC methods optimized using AQbD are published, but there are several using gradient HILIC (hydrophilic interaction chromatography) methods as well (53). Using isocratic HPLC methods, the most frequent CMPs are the aforementioned input variables $(54,55)$.

In a recent study published by Saha et al. (42) a simple, rapid and robust UPLC-MS method was developed for atazanavir sulphate using the AQbD approach. Implementing a combined technique of Ishikawa followed by FMEA risk analysis, factors (percent of organic modifier, flow rate and injection volume) that critically affect selected responses (analyte retention time, theoretical plate number, peak tailing and column) have been identified. The four factors were initially screened using a fractional factorial design for their influence on CQAs. As column temperature lacked a significant impact on method performance this was kept constant in the further stages of the method development and the remaining three factors were optimized using a Box-Behnken experimental design. After defining the method operable design region (MODR) using the 3D surface plots, method validation was carried out, with the exception of robustness testing, which in the case of experimental design is often embedded in the MODR. 


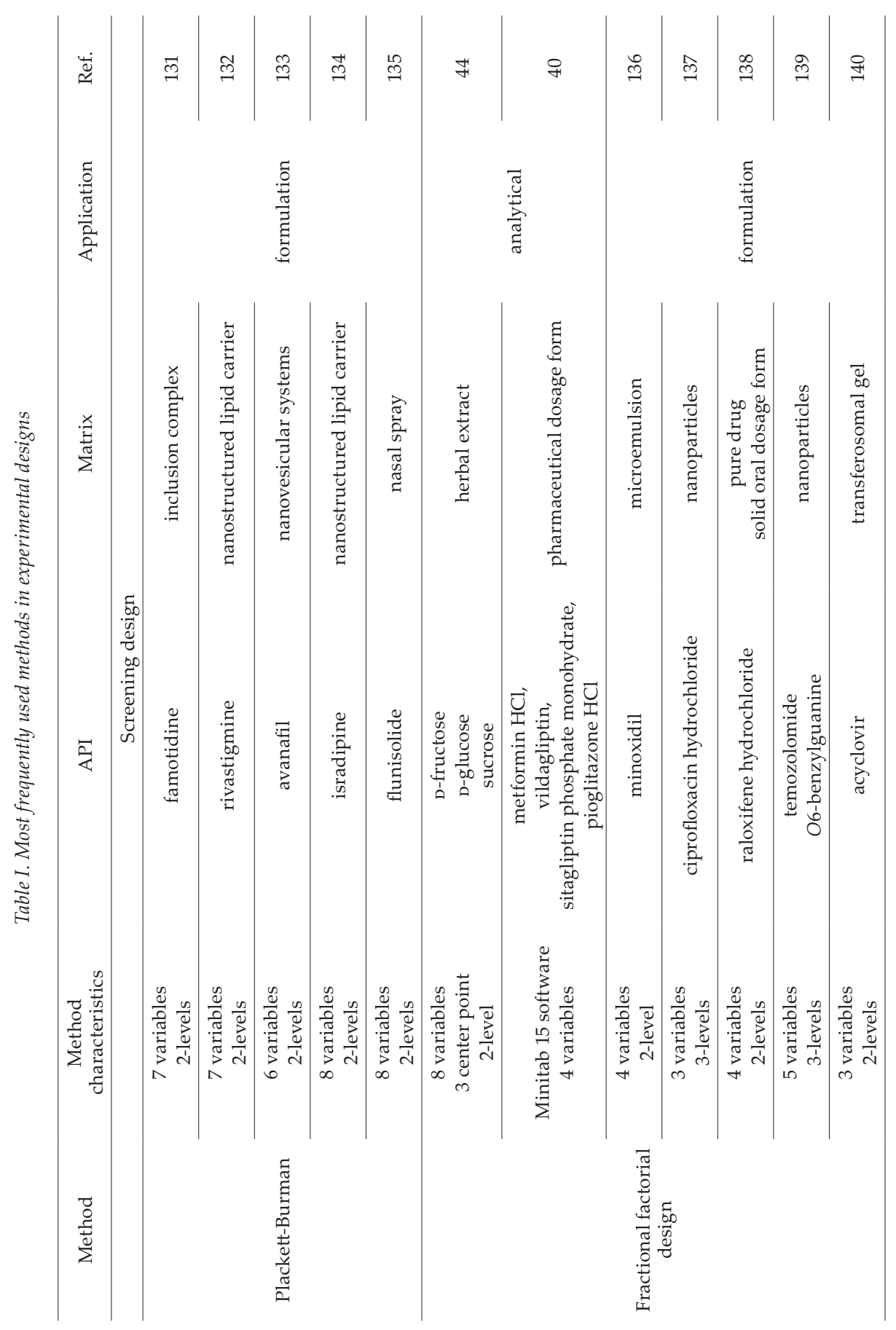




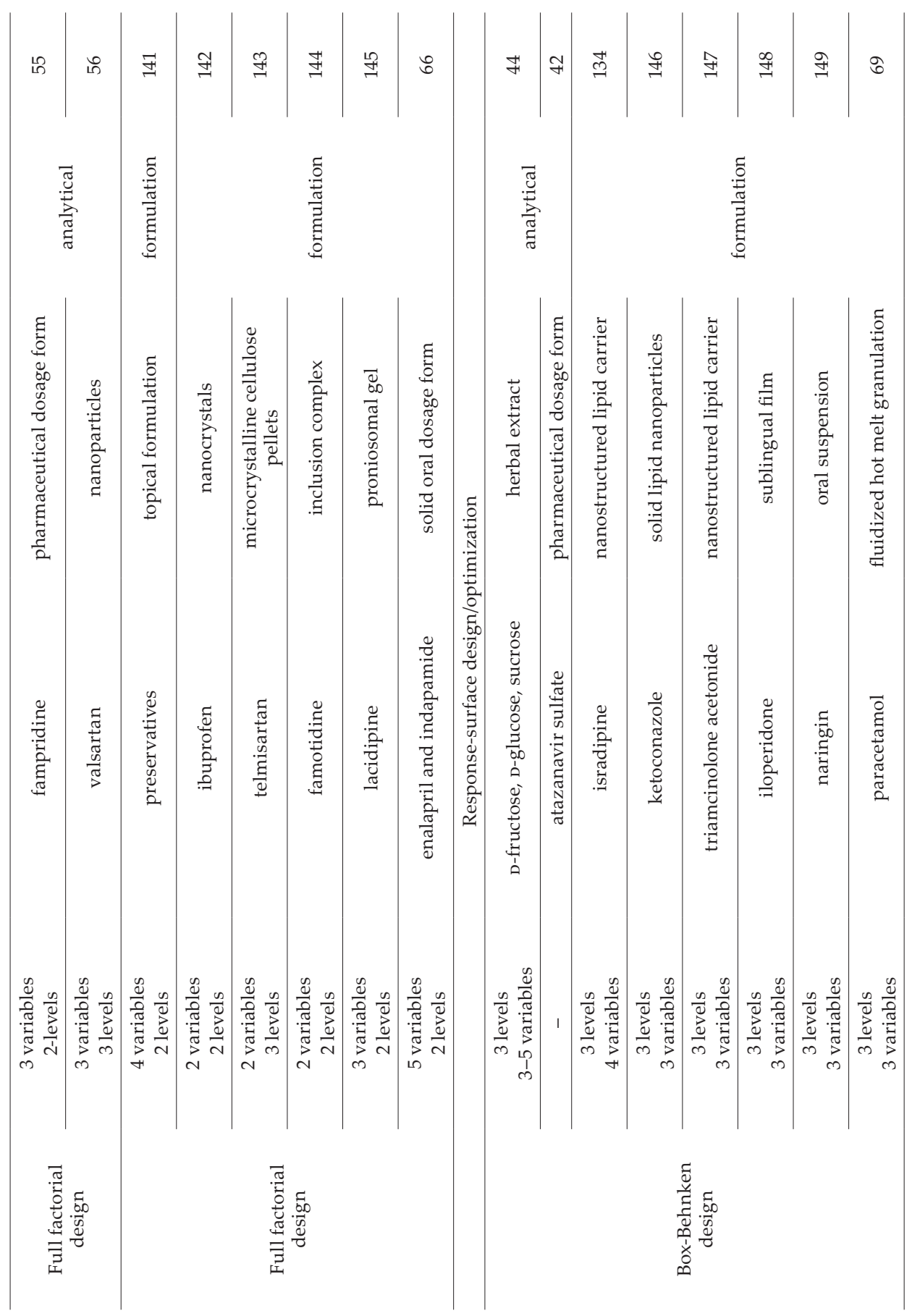




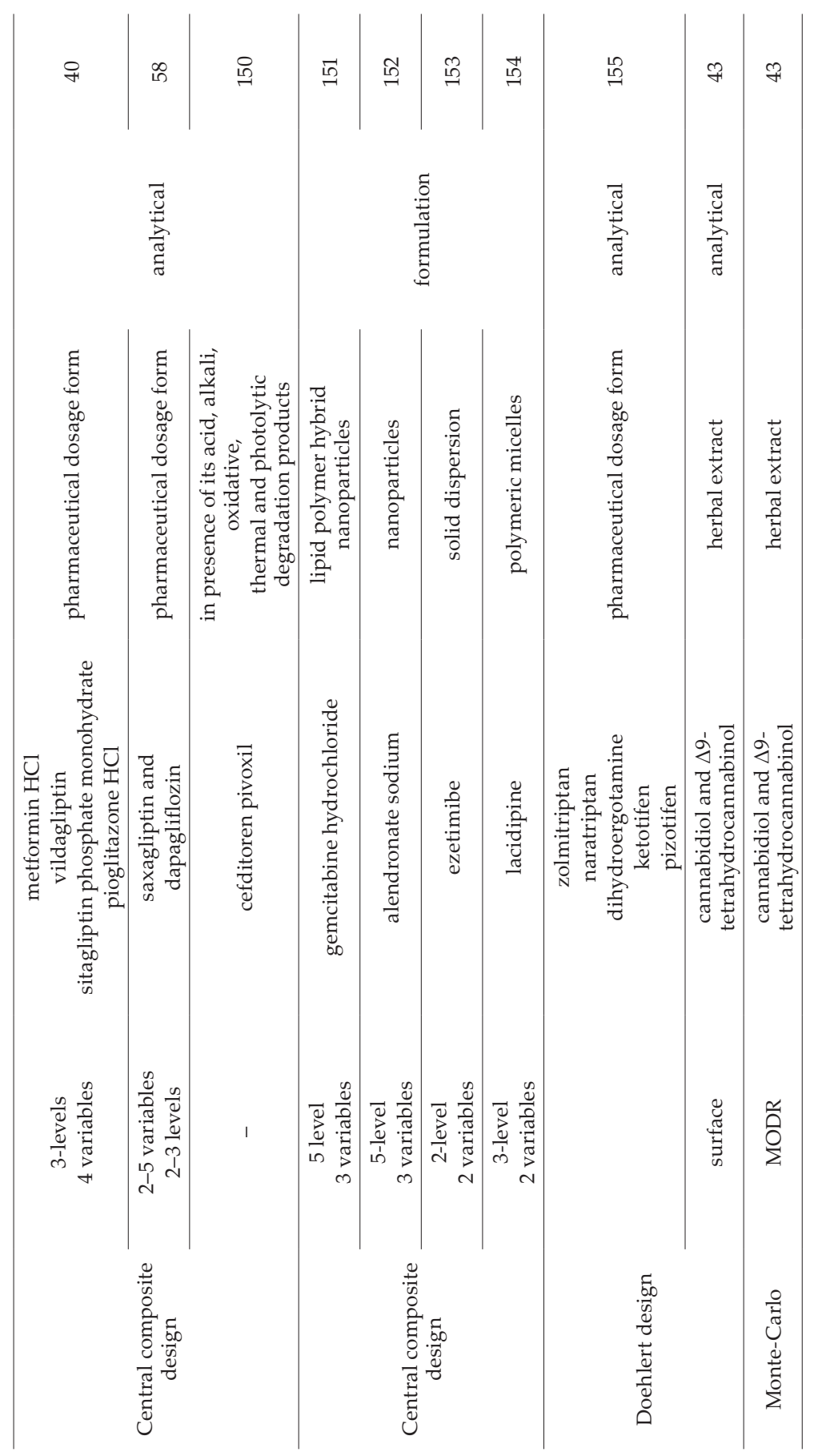


Another study, presented by Kumar et al. (56) described the development and validation of an HPLC method for the determination of valsartan in nanoparticles. DoE table was set up based on a full factorial design, including flow rate, detection wavelength and $\mathrm{pH}$ of the buffer solution as factors, noting the peak area, tailing factor and theoretical plate number as responses. The optimized parameter settings performed well in terms of predicted vs. observed response values, indicating a well-established MODR using DoE assistance. Further method validation was carried out with the optimized settings as per the requirements of the $\mathrm{ICH}$ guidelines. Once again, robustness testing was considered to be an integrated part of the defined MODR.

Similarly, we previously developed an RP-HPLC method for the quantification of fampridine from the solid oral dosage form by applying a full factorial experimental design (55). A MODR was achieved in terms of flow rate, column temperature and buffer proportion in the mobile phase, for chromatographic system CQAs (retention time, theoretical plate number and tailing factor). In addition, during the validation phase, method robustness was tested using a Plackett-Burman experimental design, in order to show that small modifications in parameter settings do not influence in a significant way the selected responses. This observation is based on markedly lower goodness of fit and goodness of predictability values when compared to those obtained during the screening type experimental design. Robustness testing through a Plackett-Burman model was also carried out by Maškovic et al. (57) for perindopril tert-butylamine in the presence of its related impurities. Through DoE the non-significant intervals for seven factors (column type, acetonitrile content, temperature, mobile phase $\mathrm{pH}$, potassium dihydrogen phosphate concentration, flow rate and wavelength) were obtained; furthermore, system-suitability tests were performed and adjacent acceptance range was defined in certain cases.

Analysing the most frequently defined CMAs, these depend on the determined ATP: the resolution of two peaks, if impurities are measured beside an active pharmaceutical ingredient or structurally similar compounds $(40,53,54,58)$, analytical sensitivity and analysis time if the analytes are measured in a complex matrix (44).

Selecting the suitable DoE tool depends on the aim of the study (screening or optimization), the number of variables and the knowledge on the impact the input variables have on results (if it is available).

(iv) Definition of a method operable design region (MODR). - Another important step in method development is defining MODR, also known as analytical design space. Design space is defined as the "multidimensional combination and interaction of input variables that have been demonstrated to provide assurance of quality" (3). MODR is the region of robustness, where the effect of CMPs (input variables) on CMAs meets the desired analytical parameters drafted by ATPs (59). Determination of MODR during the method development phase can substitute the robustness testing at the end of the method validation (42). The importance of the design space is highlighted when transferring methods between laboratories; additional validation steps are required only if the condition is moved out of the design space. For this purpose, two complementary design spaces should be determined: column design space and eluent (and other variables) design space (60), but in many cases only the second one is tested.

Various DoE techniques (Box-Behnken design, fractional factorial design, etc.) can be used for robustness testing, small changes in CMPs (flow rate, organic modifier percentage, 
column temperature, wavelength, etc.) are tested, to evaluate if they have significant effects on CMAs $(40,61)$.

In order to better visualize the effects of the input variables (such as $\mathrm{pH}$, temperature, flow rate, etc.) on CMAs, response surface analysis (2D or 3D response surface plot) can be applied, which shows the complex, non-linear interactions between the studied factors on quality performances $(42,61)$. A general workflow presenting the steps of AQbD is illustrated in Scheme $2(28,62,63)$.

Proper analytical method development assisted by computational tools is of utmost importance in modern pharmaceutical research and development as it contributes to the endowment of proper manufacturing technology and simultaneously represents the mirror of compositional or technological modifications encountered during formulation optimization.

Quality-by-design in pharmaceutical manufacturing. - Over the past decade, the pharmaceutical manufacturing processes have started to be based extensively on the principles of quality-by-design, as the standards of international authorities have greatly risen. Today, both RA and DoE are widely used to assure product quality and process ruggedness during the manufacturing of solid and liquid pharmaceutical dosage forms.

The main steps to be considered during pharmaceutical QbD are quasi-similar to those described in the previous section. First, the definition of QTPPs has to be performed, which might be based on empirical considerations, or in the case of generic product development, on the physical and in-process control characterization of the original product. In this context, QTPPs will establish the definition of the CQAs, which need to be controlled

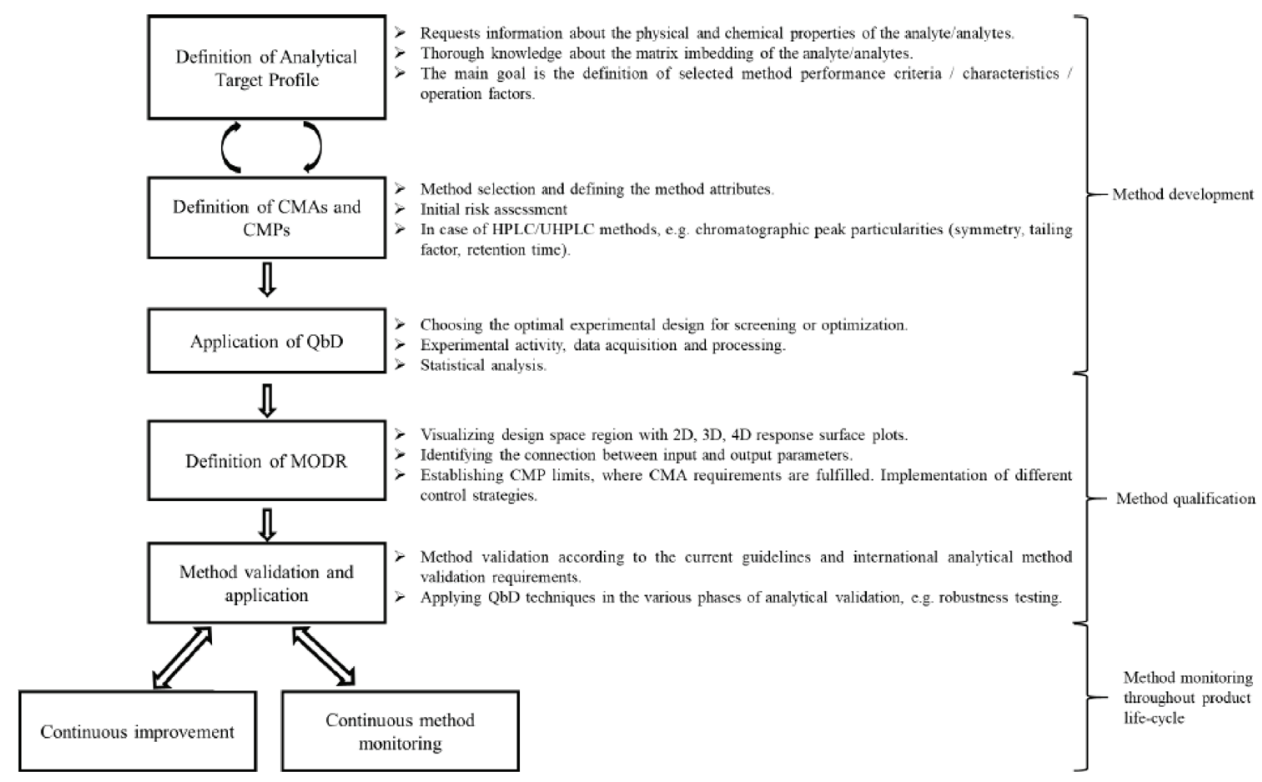

Scheme 2 
by implementing a well-established control strategy based on RA and experimental design, while taking into consideration the CMAs and CPPs of the pharmaceutical formulation and manufacturing technology, resp. $(8,64)$.

The QbD strategy has been successfully applied in the optimization and comprehension of different pharmaceutical technological processes. The CPPs in pharmaceutical manufacturing have multifactorial and process-related particularities. Solid oral dosage forms (e.g., tablets, capsules) are the most preferred forms of patient medication. The classical manufacturing processes imply dry blending or granulation techniques either via wet- (fluid bed granulation or high-shear mixing and granulation), or dry-routes (roller compaction) (65).

In the case of dry blending, formulation excipients, such as fillers, lubricants and disintegrants have an important impact on manufacturability and product quality. For a proper die filling during tablet compression, it is of utmost importance to obtain good powder flow properties. This can be achieved by selecting larger particle size excipients, but keeping insight that the excipients' distribution of particles is in the range of the APIs, in order to avoid material segregation and uneven dosage uniformity. During direct compression, the compression force, or turret and feeder rotation speed ratios should be considered as CPPs. Compression force, as one should expect, would define tablet hardness and API dissolution from the tablets' matrix, whereas the optimum turret and feeder rotation speed ratio define the mass uniformity. Szabo et al. (66) investigated the effect of formulation variables on the manufacturability of tablets containing a combination of enalapril maleate and indapamide. In their study, microcrystalline cellulose, croscarmellose sodium and magnesium stearate had a noticeable impact on manufacturability and in-process control of tablets, while compression force was noted as the most influential process parameter on product performance characteristics.

Generally, in wet-route manufacturing, the wet-granulation will have the greatest impact on product QTPPs, as granule density, size and moisture content will subsequently influence the compressibility behaviour of the final powder blend and, consequently, in vitro and in vivo release kinetics. A wet-granulation system has two main components: (i) the internal phase, usually comprising the active ingredient and bulking excipients and (ii) the granulation liquid, generally purified water or an aqueous solution of selected binder excipients (starch or derivatives thereof, gelatine, polyvinylpyrrolidone, etc.). Granulation liquid quantity and spray rates are common variables that should be considered for both fluid bed and high-shear mixing and granulation. In particular, during fluid bed granulation, inlet airflow and temperature, through moisture regulation and atomizing air pressures, through droplet size, will influence granule growth and compactness. In terms of high-shear mixing and granulation, impeller and chopper velocities are generally considered as CPPs. In a study performed by Rambali et al. (67) inlet airflow, inlet air temperature, spray rate, inlet air humidity and powder bed moisture content were selected as process variables and powder rheology characteristics were noted as responses. Similarly, Djuris et al. (68) have identified the binder addition rate, airflow and inlet air temperature as CPPs. In such systems, the droplet size will affect granule growth and density, and usually spray rate will be the main influencing process parameter. Nevertheless, a constant and controlled granule growth is the main goal, and in order to achieve this, all previously enumerated parameters should be carefully monitored throughout the develop- 
ment. This evolution is characterized by moisture content, which could be effectively monitored in-line, by proper PAT devices, e.g., NIR spectroscopy.

Besides solid oral dosage form development and optimization, DoE is vastly used in several other pharmaceutical formulation techniques, such as hot melt granulation $(69,70)$, liquid dosage forms $(71,72)$, liposome formulations $(73,74)$ and nanostructured lipid carriers (75).

As quality demands and challenges arise in the pharmaceutical industry, several complementary software-aided tools gain more and more attention in order to assure product performance, among which artificial neural networks and multivariate data analysis occupy a prominent place.

\section{APPLICATION OF ARTIFICIAL NEURAL NETWORKS AND MULTIVARIATE DATA ANALYSIS AS COMPLEMENTARY TOOLS IN PHARMACEUTICAL DEVELOPMENT}

Artificial neural networks. - An artificial neural network (ANN) is a computational model, which mimics the learning and generalization capabilities of the human brain by processing diverse input data and utilizing previous information or training to produce relevant output. ANNs are able to model complex non-linear systems, in which the connections between variable parameters are unknown $(76,77)$. ANNs consist of processing units, which form three interconnected layers (input, hidden, output), so the output of one serves as input for the next. The input layer is formed by processing units that take up the information; the hidden layer is responsible for the processing and analysis of the input, which is then transferred to the output layer (Fig. 2). Supervised or unsupervised training can be used after structuring the network with randomly chosen weights. During supervised training, actual and anticipated outputs are provided, then compared to calculate errors, causing the system to adjust weights and reduce the value of error $(78,79)$. Training

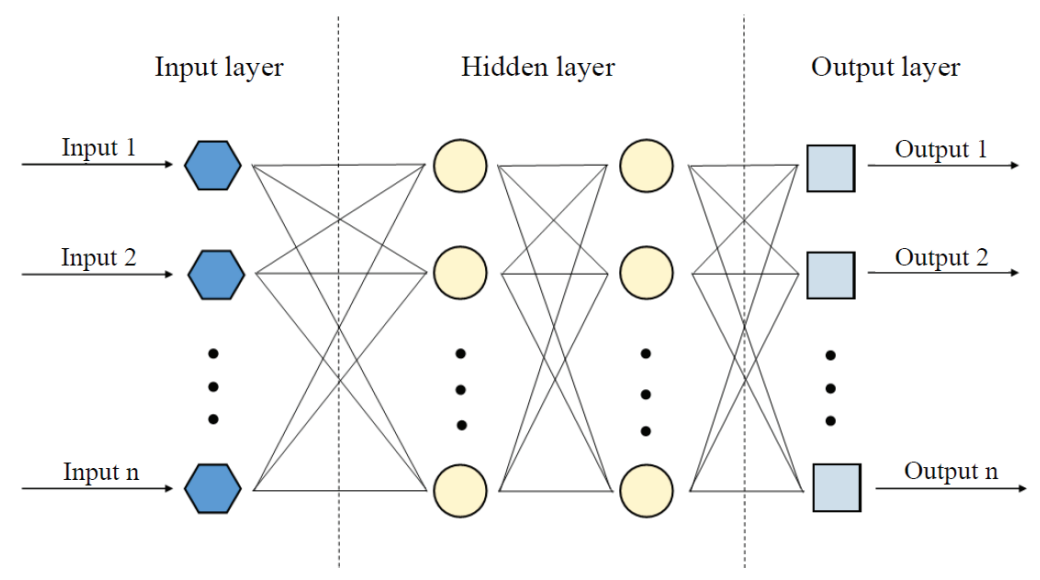

Fig. 2. Artificial neural network model. The processing units of each layer are interconnected. The input layer receives the input signals, which are passed to the hidden layer and finally to the output layer through weighted connections. 
is followed by a validation test using independent validation data, set in order to determine the capability of the model to predict correct output from unknown input data (80).

Deep neural networks (DNN) are feed-forward neural networks (FFNN) with multiple hidden layers in which information only travels forward. While shallow neural networks with a single hidden layer are faster to train and can run on machines with smaller resources, DNNs are able to model mapping with higher complexity and can be as effective as shallow neural networks when their parameters are optimized (81-83). In contrast to FFNNs, recurrent neural networks (RNN) have feedback loops, which allow signals to travel backward to previous parts of the network $(84,85)$. As a result, it allows information to persist and has the built-in capability of modelling sequential data, therefore, it is applied in model predictive control (MPC) $(86,87)$.

Pharmaceutical applications of ANNs include drug design, optimizing preformulation and formulation of various dosage forms, modelling in vitro-in vivo correlations, interpreting analytical data, determining the relationship between the chemical structure and biological activity of a substance (quantitative structure-activity relationship) as well as pharmacotoxicological, pharmacokinetic and pharmacodynamic studies $(76,88)$. ANN has been adopted to predict the release rate from lignin-containing aspirin tablets (89) and the characteristics of buccoadhesive pharmaceutical wafers containing loratadine (90). ANN models have been used to predict the disintegration time, friability and tablet hardness of orally disintegrating ondansetron tablets (91). Han et al. (92) compared the accuracy of the disintegration time of orally disintegrating tablet formulations predicted with ANN and DNN techniques; DNN proved to be significantly better at providing real unknown data when compared to ANN. Ilić et al. (93) developed in vitro-in vivo correlation models based on ANN analysis in the case of nifedipine osmotic release tablets.

Furthermore, ANNs have been successfully used for in vitro release kinetics prediction of active substances from hydrophilic matrix formulations $(94,95)$. Moreover, in the past years, ANNs were combined with various computational tools, e.g., gene algorithms, for the evaluation of the pharmacokinetic parameters of alkaloid drugs (96). RNN has been applied in MPC of CQAs in continuous pharmaceutical manufacturing with favourable results (97).

Multivariate data analysis. - Multivariate data analysis (MVDA) is a statistical technique used to simultaneously analyse data sets with several variables often correlated with each other. The term chemometrics is often used when relevant chemical information is extracted from the experimental data. In addition to data analysis, the aim of MVDA is sample classification and prediction of response variables (98-100). This is achieved by a variety of multivariate methods, with principal component analysis (PCA) and partial least squares (PLS) being the most commonly used techniques for analysing continuous processes (101).

PCA reduces the number of variables in a process while retaining as much variability as possible. Correlated original variables are represented by principal components (PC) that explain the main variance in the data and are uncorrelated with each other. The first PC is the linear combination of the original variables, which accounts for maximum variability in the data. The second and subsequent PC capture as much of the remaining variability as possible. The resulting data set is easier to analyse, visualize and interpret $(102,103)$. PLS is also used to reduce the number of variables through linear transformations, however, these variables are calculated to maximize the covariance between different sets of variables (independent or exogenous and dependent data sets) using the fewest dimensions. PLS is 
especially useful in the case of small datasets, missing values and multicollinearity between predictors $(68,104)$. Both PCA and PLA are often combined with spectrophotometry in order to build simple, fast, efficient, low cost and non-destructive chemometric models that do not generate chemical waste.

PCA was implemented in order to examine the deformation of pharmaceutical powders during powder compaction (105) and to select the most important variables for developing a solid self-emulsifying drug delivery system for docetaxel (106). A combination of PCA and attenuated total reflectance Fourier-transform infrared spectroscopy (ATR-FTIR) was used to identify the polymorphic forms of mebendazole in tablets (107).

PLS was applied for the simultaneous quantitative determination of paracetamol and tramadol in paracetamol-tramadol tablets (108) and for the analysis of a binary mixture tablet containing bisoprolol fumarate and hydrochlorothiazide (109) from UV spectrophotometric data. A method based on diffuse reflectance, middle infrared spectroscopy and PLS was used for simultaneous quantification of artesunate and mefloquine in tablets (110).

Both PCA and PLS have been useful in selecting suitable and desired CQAs of nanostructured lipid carriers (111). Ali et al. (112) built a chemometric model by combining multivariate data analysis tools (PCA and PLS) and vibrational spectroscopy (FTIR/NIR) method for quantification of carbamazepine dihydrate in the formulation. Arabzadeh et al. (113) developed a UV spectrophotometric analysis method based on ANN, PLS and principal component regression (PCR) for simultaneous determination of emtricitabine and tenofovir alafenamide fumarate in commercial HIV drug. Each method showed enough accuracy when compared to the reference method (HPLC), however, the ANN and the PLS method worked better than PCR. Combined use of PCA and ANN was developed for the multicomponent determination of four drugs from a complex pharmaceutical mixture using UV spectroscopy, which gave the best results with a shorter convergence speed when compared to ANN (114).

Process analytical technology (and major process analyzers applied) in quality-by-design. - Process analytical technology (PAT), is a relatively new concept to the pharmaceutical industry, yet it has been already known previously as process analytical chemistry (PAC) in other industries. The FDA released the guidance on PAT in final form in September 2004 as a framework for innovative pharmaceutical development, manufacturing and quality assurance. The aim of the document was to enhance process understanding, leading to improved control of the manufacturing process. While the ultimate goal of $\mathrm{QbD}$ is to build quality into products by design, PAT plays a crucial role in its accomplishment. PAT tools and principles represent the means to achieve the aims of $\mathrm{QbD}$. The application of PAT is encouraged by FDA representatives (115).

PAT is composed of multivariate (chemical, physical, microbiological and mathematical) methods for material and process analysis, containing also process modelling and enhanced control strategies in order to enhance product quality and process efficiency. PAT has involved concomitant with the decrease of measurement systems' cost, developments in microsystem technology and the explosive increase of computational power experienced in the last decade (116).

Process analysers are the heart of PAT tools permitting real-time process monitoring and control. They generate the necessary data from which valuable information could be extracted about processes and products. Unlike conventional systems which provide uni- 
variate measurements, the PAT process analysers furnish multivariate information in conjunction with biological, physical, and chemical attributes of the starting materials, intermediates and finished drug products. The major considerations which should be taken into account when selecting process analysers into the technological flux are the following: the ability to properly monitor the process and/or product properties, defining measurement conditions, proper location of the analyser and the validation of performance (117).

The vast majority of applications in the pharmaceutical industry rely on two of the vibrational spectroscopic techniques: near-infrared spectroscopy (NIR), followed by Raman spectroscopy. These methods allow rapid and non-destructive measurements providing versatile and multivariate information and have been implemented in the common batch unit operations of the solid dosage form development and manufacturing processes for monitoring critical aspects. Besides the use of the NIR and Raman spectrometers in the application as in-line, at-line or on-line analyzers in many studies these are used as off-line tools. Further standard operations are imaging (for extracting morphological features) and mainly in the case of liquids UV-VIS analysis. However, other technologies are quickly catching up and increasing the repository of possible PAT tools (116).

As published by Helešicová et al. (118) in the case of topochemical mapping of pharmaceutical tablets from microtome slices with Raman microscopy the experimental setup is of great importance and depending on the resolution required, the selection of proper measurement type plays also a crucial role. Among others, the study highlights the use of advanced multivariate chemometric methods (as PCA and SIMCA) and encourages the application of different evaluation approaches in order to improve the reliability of gathered information.

In a study presented by Mitsutake et al. (119) the Raman mapping in association with three different chemometric methods [(PCA, multivariate curve resolution alternating least square (MCR-ALS) and independent component analysis (ICA)] was applied in the investigation of the spatial distribution of components in case of semi-solid pharmaceutical systems and predicting their stability and possible physical transformations (118).

In a recent study, Nomura et al. (120) demonstrated the utility of a non-contact lowfrequency Raman probe in the real-time monitorization of polymorph conversion in the course of high-shear wet granulation. Four model drugs (indomethacin, acetaminophen, theophylline and caffeine) were involved in the study and were subjected to aqueous wet granulation in the presence of broadly used pharmaceutical excipients. The study also extended to the practical detection limit of API content, monitoring the hydrate transition of crystalline drugs, dissociation monitoring of co-crystals as well as the feasibility study of monitoring of commercial formulations. The obtained results were confirmed by X-ray powder diffraction analysis.

In a recent study, Gavan et al. (121) monitored the moisture content of the fluidised-bed step by the means of micro-NIR PAT-U in order to improve the supervision and control over the process. It was demonstrated that NIR analysis is a reliable PAT tool and might substitute the currently broadly applied loss on drying measurement based on a gravimetric method (119).

Casian et al. (122) validated and compared NIR and Raman spectroscopic methods for the characterization of fixed-dose combination tablets. They managed to establish highly predictive computational models for quantification of API by NIR-transmission and 
Raman-reflectance as well as NIR transmittance for tensile strength in case of amlodipine and valsartan-containing tablets.

Among the emerging techniques that started to be implemented in the PAT, terahertz (THz) spectroscopy should be mentioned which uses THz pulses generated and detected by short-pulsed lasers. The pulse widths are ranging from 10-100 femtoseconds and are characteristic to terahertz spectroscopy in comparison to conventional far-IR investigations. As the majority of crystalline solids exhibit characteristic lattice vibrations in this specific spectral range, this technique could be applied in the investigation of intermolecular interactions of molecular crystals. These characteristics might be explored in polymorph screening, mapping of chemical compositions, determining tablet density and for characterizing the film-coatings among others $(123,124)$.

Another promising spectroscopic method which should be noticed in conjunction with PAT is nuclear magnetic resonance (NMR) spectroscopy. This method was already applied for the quality control and authenticity of pharmaceutical products and its importance will surely increase in the upcoming period (125). More and more techniques are emerging and trying to find their place in the PAT approach of product development and manufacturing, therefore, without being exhaustive, novel methods as optical coherence tomography (OCT), acoustic emission (AE), microwave resonance (MR), and laser-induced breakdown spectroscopy (LIBS) should also be mentioned (126). In view of particle size on-line measurement essential to the proper monitorization of the widely used granulation process, the methods which have been developed and implemented as PAT tools are imaging, focused beam reflectance measurement (FBRM) and spatial filter velocimetry (SFV) (127). The last two rely on the so-called chord length distribution (CLD) approach which measures the length of the signal generated by the interaction between the laser beam and the particles analysed (126). These techniques have been successfully applied in the growth kinetics monitoring during the fluidized bed- $(128,129)$ and high-shear wet granulation processes (130).

In PAT the data collected from different locations and varying instruments have to be combined with conventional data processing into meaningful relationships. After data consolidation, this information can be used in feedback or even feed-forward control loops with different complexity. This is achieved without further testing procedures, realizing real-time release testing (RTRT). In practice this step is critical and tests are often substituted one-by-one. Via in-line, on-line or at-line measurements throughout the manufacturing process stream with enough confidence in the data, and enough accuracy in the quantitative predictions, the obtained information can be used to directly assess the quality of the products and might make attainable the real-time release (RTR) (116).

\section{CONCLUSIONS}

Assuring and controlling the quality and robustness of the methods for manufacturing pharmaceutical dosage forms has involved important changes in the past decades. Several guidelines have been imposed to identify, evaluate and control potential hazards, which might lead to undesirable out-of-specification product results and eventually a greater batch-to-batch variation. In the current view, standalone RA, or RA augmented by various in silico methods are used for the aforementioned purposes. The implementation 
and application of the computational methods for $\mathrm{QbD}$ represent one of the key future perspectives and challenges. Although at first sight the complex nature of QbD might seem to significantly increase costs and be more time-consuming when compared to the OFAT concept, the late-stage advantages during scale-up to pilot, or industrial scale is unquestionable, and represent an opportunistic approach for constant quality assurance throughout the product lifecycle. In this perspective, complementary tools and techniques, like ANN, MVDA and PAT will further augment these novel orientations in pharmaceutical $R \& D$, contributing to enhanced quality assurance in the pharmaceutical industry.

The intent of this article was to provide a general overview of possible applications for $\mathrm{QbD}$ in the pharmaceutical industry, including examples where these methods have already been successfully used and identifying relevant publications containing a detailed, mathematical approach for the presented concepts.

Acknowledgments. - László-István Bába is a recipient of a Collegium Talentum Scholarship Programme of Hungary and thanks for the support.

Acronyms, abbreviations, symbols. - AE - acoustic emission, ANN - artificial neural networks, $\mathrm{AQbD}$ - analytical quality by design, ATP - analytical target profile, CLD - chord length distribution, CMA - critical material attributes (technologic aspects)/critical method attributes (analytical aspects), CMC - chemistry, manufacturing and controls, CMP - critical method parameters, CPP - critical process parameter, CQA - critical quality attributes, DNN - deep neural networks, DoE - design of experiments (experimental design), FBRM - focused beam reflectance measurement, FFNN - feed forward neural networks, FMEA - failure mode and effects analysis, FMECA - failure mode and effects critical analysis, FTA - fault tree analysis, HACCP - hazard analysis and critical control points, HAZOP - hazard and operability study, ICA - independent component analysis, LIBS - laser induced breakdown spectroscopy, MCR-ALS - multivariate curve resolution alternating least square, MODR - method operable design region, MPC - model predictive control, MR - microwave resonance, MVDA - multivariate data analysis, OCT - optical coherence tomography, OFAT - one-factor-at-atime, PAC - process analytical chemistry, PAT - process analytical technology, PAT-U - type of apparatus used for the determination of moisture (ref. 121), PC - principal components, PCA - principal component analysis, PCR - principal component regression, PHA - preliminary hazard analysis, PLS - partial least squares, QbD - quality-by-design, QTPP - quality target product profile, RA - risk assessment, RNN - recurrent neural networks, RTR - real-time release, RTRT - real-time release testing, SIMCA - soft independent modelling of class analogies, SFV - spatial filter velocimetry.

\section{REFERENCES}

1. R. A. Lionberger, S. L. Lee, L. M. Lee, A. Raw and L. X. Yu, Quality by design: Concepts for ANDAs, AAPS J. 10 (2008) 268-276; https://doi.org/10.1208/s12248-008-9026-7

2. K. Pramod, M. Tahir, N. Charoo, S. Ansari and J. Ali, Pharmaceutical product development: A quality by design approach, Int. J. Pharm. Investig. 6 (2016) 129-138; https://doi.org/10.4103/2230-973x.187350

3. International Conference on Harmonisation of Technical Requirements for Registration of Pharmaceuticals for Human Use, ICH Harmonised Tripartite Guideline, Pharmaceutical Development, Q8(R2), Current Step 5 version, August 2009; https://www.ema.europa.eu/en/documents/scientificguideline/international-conference-harmonisation-technical-requirements-registration-pharmaceuticals-human-use_en-11.pdf; access date: October 26, 2020

4. International Conference on Harmonisation of Technical Requirements for Registration of Pharmaceuticals for Human Use, ICH Harmonised Tripartite Guideline, Quality Risk Management, Q9, Current Step 5 version, August 2009; https://www.ema.europa.eu/en/documents/scientific-guideline/ 
international-conference-harmonisation-technical-requirements-registration-pharmaceuticalshuman-use_en-11.pdf; access date: October 26, 2020

5. Y. Bouwman-Boer and L. Møller Andersen, Pharmaceutical Quality Systems, in Practical Pharmaceutics (Ed. Y. Bouwman-Boer, L. Møller Andersen and P. Le Brun), Springer Nature, Cham (Switzerland) 2015, pp. 769-796.

6. International Conference on Harmonisation of Technical Requirements for Registration of Pharmaceuticals for Human Use, Development and manufacture of drug substances (chemical entities and biotechnological/biological entities), Q11, Current Step 3 version, September 2011; https://www.ema.europa.eu/ en/documents/scientific-guideline/draft-ich-guideline-q11-development-manufacture-drug-substances-chemical-entities-biotechnological/biological-entities_en.pdf; access date: October 26, 2020

7. International Conference on Harmonisation of Technical Requirements for Registration of Pharmaceuticals for Human Use, Technical and regulatory considerations for pharmaceutical product lifecycle management, Q12, Current Step 5 version, January 2020; https:/www.ema.europa.eu/en/documents/ scientific-guideline/ich-guideline-q12-technical-regulatory-considerations-pharmaceutical-product-lifecycle-management_en.pdf; access date: October 26, 2020

8. L. X. Yu, G. Amidon, M. A. Khan, S. W. Hoag, J. Polli, G. K. Raju and J. Woodcock, Understanding pharmaceutical quality by design, AAPS J. 16 (2014) 771-783; https://doi.org/10.1208/s12248-014-9598-3

9. International Conference on Harmonisation of Technical Requirements for Registration of Pharmaceuticals for Human Use, Continuous Manufacturing of Drug Substances and Drug Products, Q13, Final Concept Paper, November 2018; https://database.ich.org/sites/default/files/Q13_EWG_Concept_Paper.pdf; access date: October 26, 2020

10. International Conference on Harmonisation of Technical Requirements for Registration of Pharmaceuticals for Human Use, Analytical Procedure Development and Revision of Q2(R1) Analytical Validation Q14, Final Concept Paper, November 2018; https://database.ich.org/sites/default/files/Q2R2-Q14_ EWG_Concept_Paper.pdf; access date: October 26, 2020

11. J. Cook, M. T. Cruañes, M. Gupta, S. Riley and J. Crison, Quality-by-design: Are we there yet?, AAPS PharmSciTech 15 (2014) 140-148; https://doi.org/10.1208/s12249-013-0043-1

12. H. B. Grangeia, C. Silva, S. P. Simões and M. S. Reis, Quality by design in pharmaceutical manufacturing: A systematic review of current status, challenges and future perspectives, Eur. J. Pharm. Biopharm. 147 (2020) 19-37; https://doi.org/10.1016/j.ejpb.2019.12.007

13. A. Aloqaily, Identification of Hazards Associated with Pipelines, in Cross-Country Pipeline Risk Assessments and Mitigation Strategies (Ed. A. Aloqaily), Elsevier, Amsterdam 2018, pp. 13-40.

14. J. W. Vincoli, Preliminary Hazard Analysis, in Basic Guideline to System. Safety (Ed. J. W. Voncoli), $3^{\text {rd }}$ Edition, Wiley, Hoboken (NJ) 2014, pp. 71-91.

15. A. F. Molland, Marine Safety, in The Maritime Engineering Reference Book (Ed. A. F. Molland), Elsevier, Amsterdam 2008, pp. 784-875.

16. G. Ilie and C. Ciocoiu, Application of fishbone diagram to determine the risk of an event with multiple causes, Manag. Res. Pract. 2 (2010) 1-20.

17. M. A. Barsalou, The Quality Improvement Field Guide: Achieving and Maintaining Value in Your Organization, Taylor \& Francis, Oxfordshire 2016.

18. T. Aven, Risk assessment and risk management: Review of recent advances on their foundation, Eur. J. Oper. Res. 253 (2016) 1-13; https://doi.org/10.1016/j.ejor.2015.12.023

19. J. F. van Leeuwen, M. J. Nauta, D. de Kaste, Y. M. C. F. Odekerken-Rombouts, M. T. Oldenhof, M. J. Vredenbregt and D. M. Barends, Risk analysis by FMEA as an element of analytical validation, J. Pharm. Biomed. Anal. 50 (2009) 1085-1087; https://doi.org/10.1016/j.jpba.2009.06.049

20. M. T. Oldenhof, J. F. van Leeuwen, M. J. Nauta, D. de Kaste, Y. M. C. F. Odekerken-Rombouts, M. J. Vredenbregt, M. Weda and D. M. Barends, Consistency of FMEA used in the validation of analytical procedures, J. Pharm. Biomed. Anal. 54 (2011) 592-595; https://doi.org/10.1016/j.jpba.2010.09.024 
21. R. Fahmy, R. Kona, R. Dandu, W. Xie, G. Claycamp and S. W. Hoag, Quality by design I: Application of failure mode effect analysis (FMEA) and Plackett-Burman design of experiments in the identification of "main factors" in the formulation and process design space for roller-compacted ciprofloxacin hydrochloride immediate, AAPS PharmSciTech 13 (2012) 1243-1254; https://doi.org/10.1208/ s12249-012-9844-x

22. N. A. Wessiani and F. Yoshio, Failure mode effect analysis and fault tree analysis as a combined methodology in risk management, IOP Conference Series: Materials Science and Engeneering. 337 (2018) Article ID 012033 (11 pages); https://doi.org/10.1088/1757-899X/337/1/012033

23. M.W.Averett, Fault Tree Analysis, RiskAnalysis 8(1988)463-464;https://doi.org/10.1111/j.1539-6924.1988. tb00510.x

24. S. Iurian, L. Turdean and I. Tomuta, Risk assessment and experimental design in the development of a prolonged release drug delivery system with paliperidone, Drug Des. Devel. Ther. 11 (2017) 733-746; https://doi.org/10.2147/DDDT.S125323

25. S. M. Mishra and B. D. Rohera, An integrated, quality by design (QbD) approach for design, development and optimization of orally disintegrating tablet formulation of carbamazepine, Pharm. Dev. Technol. 22 (2017) 889-903; https://doi.org/10.1080/10837450.2016.1199566

26. X. Zhang and C. Hu, Application of quality by design concept to develop a dual gradient elution stability-indicating method for cloxacillin forced degradation studies using combined mixtureprocess variable models, J. Chromatogr. A 1514 (2017) 44-53; https://doi.org/10.1016/j.chroma.2017.07.062

27. G. L. Reid, G. Cheng, D. T. Fortin, J. W. Harwood, J. E. Morgado, J. Wang and G. Xue, Reversed-phase liquid chromatographic method development in an analytical quality by design framework, J. Liq. Chromatogr. Relat. Technol. 36 (2013) 2612-2638; https://doi.org/10.1080/10826076.2013.765457

28. P. Borman, M. Chatfield, P. Nethercote, D. Thompson and K. Truman, The application of quality by design to analytical methods, Pharm. Technol. 31 (2007) 142-152.

29. B. Pasquini, S. Orlandini, M. Villar-Navarro, C. Caprini, M. Del Bubba, M. Douša, A. Giuffrida, R. Gotti and S. Furlanetto, Chiral capillary zone electrophoresis in enantioseparation and analysis of cinacalcet impurities: Use of Quality by Design principles in method development, J. Chromatogr. A 1568 (2018) 205-213; https://doi.org/10.1016/j.chroma.2018.07.021

30. S. Krait and G. K. E. Scriba, Quality by design-assisted development of a capillary electrophoresis method for the chiral purity determination of dexmedetomidine, Electrophoresis 39 (2018) 2575-2580; https://doi.org/10.1002/elps.201800100

31. L. Zhou, J. M. Socha, F. G. Vogt, S. Chen and A. S. Kord, A systematic method development strategy for water determinations in drug substance using Karl Fischer titrations, Am. Pharm. Rev. 13 (2010) 74-84.

32. L. Zhou, F. G. Vogt, P. A. Overstreet, J. T. Dougherty, J. S. Clawson and A. S. Kord, A systematic method development strategy for quantitative color measurement in drug substances, starting materials, and synthetic intermediates, J. Pharm. Innov. 6 (2011) 217-231; https://doi.org/10.1007/s12247011-9115-5

33. F. G. Vogt and A. S. Kord, Development of quality-by-design analytical methods, J. Pharm. Sci. 100 (2011) 797-812; https://doi.org/10.1002/jps.22325

34. R. Peraman, K. Bhadraya and Y. P. Reddy, Analytical quality by design: A tool for regulatory flexibility and robust analytics, Int. J. Anal. Chem. 2015 (2015) Article ID 868727 (9 pages); https://doi. org/10.1155/2015/868727

35. P. Jackson, P. Borman, C. Campa, M. Chatfield, M. Godfrey, P. Hamilton, W. Hoyer, F. Norelli, R. Orr and T. Schofield, Using the analytical target profile to drive the analytical method lifecycle, Anal. Chem. 91 (2019) 2577-2585; https://doi.org/10.1021/acs.analchem.8b04596 
36. M. A. Gad, S. M. Amer, H. E. Zaazaa and S. A. Hassan, Strategies for stabilizing formulation and QbD assisted development of robust stability indicating method of azilsartan medoxomil/chlorthalidone, J. Pharm. Biomed. Anal. 178 (2020) Article ID 112910; https://doi.org/10.1016/j.jpba.2019.112910

37. B. Kovács, L. K. Kántor, M. D. Croitoru, É. K. Kelemen, M. Obreja, E. E. Nagy, B. Székely-Szentmiklósi and Á. Gyéresi, Reversed phase HPLC for strontium ranelate: Method development and validation applying experimental design, Acta Pharm. 68 (2018) 171-183; https://doi.org/10.2478/acph-20180019

38. A. Dispas, H. T. Avohou, P. Lebrun, P. Hubert and C. Hubert, 'Quality by Design' approach for the analysis of impurities in pharmaceutical drug products and drug substances, TrAC - Trends Anal. Chem. 101 (2018) 24-33; https://doi.org/10.1016/j.trac.2017.10.028

39. P. Ramalingam and B. Jahnavi, QbD Considerations for Analytical Development, in Pharmaceutical Quality by Design - Principles and Applications (Ed. S. Beg and S. Hasnain), Elsevier, Amsterdam 2019, pp. 77-108.

40. R. M. Ahmed, A. Ibrahim, A. E. El-Gendy and G. M. Hadad, Implementing a Quality by Design approach in chromatographic determination of some antidiabetic drugs, SF J. Pharm. Anal. Chem. 1 (2018) Article ID 1001 (9 pages).

41. T. Tome, N. Žigart, Z. Časar and A. Obreza, Development and optimization of liquid chromatography analytical methods by using AQbD principles: Overview and recent advances, Org. Process Res. Dev. 23 (2019) 1784-1802; https://doi.org/10.1021/acs.oprd.9b00238

42. C. Saha, N. V. Gupta and R. S. Chandan, Development and validation of a UPLC-MS method for determination of atazanavir sulfate by the "analytical quality by design" approach, Acta Pharm. 70 (2020) 17-33; https://doi.org/10.2478/acph-2020-0008

43. R. Deidda, H. T. Avohou, R. Baronti, P. L. Davolio, B. Pasquini, M. Del Bubba, C. Hubert, P. Hubert, S. Orlandini and S. Furlanetto, Analytical quality by design: Development and control strategy for a LC method to evaluate the cannabinoids content in cannabis olive oil extracts, J. Pharm. Biomed. Anal. 166 (2019) 326-335; https://doi.org/10.1016/j.jpba.2019.01.032

44. J. Shao, W. Cao, H. Qu, J. Pan and X. Gong, A novel quality by design approach for developing an HPLC method to analyze herbal extracts: A case study of sugar content analysis, PLoS One 13 (2018); e0198515; https://doi.org/10.1371/journal.pone.0198515

45. A. S. K. Sankar, P. Shanmugasundaram and R. Velayudham, Quality by design-applied liquid chromatography-tandem mass spectrometry determination of enzalutamide anti-prostate cancer therapy drug in spiked plasma samples, Anal. Chem. Insights 12 (2017) 1-11; https://doi. org/10.1177/1177390117726776

46. M. Deepa, K. R. Reddy and S. V. Satyanarayana, A review on quality by design approach for analytical method development, J. Pharm. Res. 11 (2017) 272-277.

47. V. Das, B. Bhairav and R. B. Saudagar, Quality by design approaches to analytical method development, Res. J. Pharm. Technol. 10 (2017) 3188-3194; https://doi.org/10.5958/0974-360X.2017.00567.4

48. J. R. Wagner, Jr., E. M. Mount III and H. F. Giles, Jr., Extrusion: The Definitive Processing Guide and Handbook, $2^{\text {nd }}$ Edition, Elsevier, Amsterdam 2013.

49. I. M. Fukuda, C. F. F. Pinto, C. dos Santos Moreira, A. M. Saviano and F. R. Lourenço, Design of experiments (DoE) applied to pharmaceutical and analytical quality by design (QbD), Braz. J. Pharm. Sci. 54 (2018) e01006 (16 pages); https://doi.org/10.1590/s2175-97902018000001006

50. C. Croarkin and P. Tobias, NIST/SEMATECH e-handbook of Statistical Methods, http://www.itl.nist. gov/div898/handbook; last access date January 31, 2020

51. P. K. Sahu, N. R. Ramisetti, T. Cecchi, S. Swain, C. S. Patro and J. Panda, An overview of experimental designs in HPLC method development and validation, J. Pharm. Biomed. Anal. 147 (2018) 590-611; https://doi.org/10.1016/j.jpba.2017.05.006 
52. B. Sylvester, L. Tefas, L. Vlase, I. Tomuță and A. Porfire, A Quality by Design (QbD) approach to the development of a gradient high-performance liquid chromatography for the simultaneous assay of curcuminoids and doxorubicin from long-circulating liposomes, J. Pharm. Biomed. Anal. 158 (2018) 395-404; https://doi.org/10.1016/j.jpba.2018.06.018

53. A. Tumpa, S. Mišković, Z. Stanimirović, B. Jančić-Stojanović and M. Medenica, Modeling of HILIC retention behavior with theoretical models and new spline interpolation technique, J. Chemom. 31 (2017) e2910; https://doi.org/10.1002/cem.2910

54. E. Ferencz, B. Kovács, F. Boda, M. Foroughbakhshfasaei, É. K. Kelemen, G. Tóth and Z. I. Szabó, Simultaneous determination of chiral and achiral impurities of ivabradine on a cellulose tris(3-chloro-4-methylphenylcarbamate) chiral column using polar organic mode, J. Pharm. Biomed. Anal. 177 (2020) Article ID 112851; https://doi.org/10.1016/j.jpba.2019.112851

55. B. Kovács, F. Boda, I. Fülöp, I. Székely-Szentmiklósi, É. K. Kelemen, B. Kovács-Deák and B. SzékelySzentmiklósi, HPLC method development for fampridine using Analytical Quality by Design approach, Acta Pharm. 70 (2020) 465-482; https://doi.org/10.2478/acph-2020-0036

56. L. Kumar, M. S. Reddy, R. S. Managuli and G. Pai K., Full factorial design for optimization, development and validation of HPLC method to determine valsartan in nanoparticles, Saudi Pharm. J. 23 (2015) 549-555; https://doi.org/10.1016/j.jsps.2015.02.001

57. M. Mašković, B. Jančić-Stojanović, A. Malenović, D. Ivanović and M. Medenica, Assessment of liquid chromatographic method robustness by use of Plackett-Burman design, Acta Chromatogr. 22 (2010) 281-296; https://doi.org/10.1556/AChrom.22.2010.2.10

58. A. Gundala, K. Prasad and B. Koganti, Application of quality by design approach in RP-HPLC method development for simultaneous estimation of saxagliptin and dapagliflozin in tablet dosage form, Braz. J. Pharm. Sci. 55 (2019) e18129; https://doi.org/10.1590/s2175-97902019000218129

59. K. Gupta, Analytical Quality by Design: A mini review, Biomed. J. Sci. Tech. Res. 1 (2017) 1555-1559; https://doi.org/10.26717/bjstr.2017.01.000484

60. K. E. Monks, H. J. Rieger and I. Molnár, Expanding the term “Design Space" in high performance liquid chromatography (I), J. Pharm. Biomed. Anal. 56 (2011) 874-879; https://doi.org/10.1016/j. jpba.2011.04.015

61. P. Bhatt, M. Saquib Hasnain, A. K. Nayak, B. Hassan and S. Beg, Development and validation of QbD-driven bioanalytical LC-MS/MS method for the quantification of paracetamol and diclofenac in human plasma, Anal. Chem. Lett. 8 (2018) 677-691; https://doi.org/10.1080/22297928.2018.1429305

62. N. V. V. S. S. Raman, U. R. Mallu and H. R. Bapatu, Analytical Quality by Design approach to test method development and validation in drug substance manufacturing, J. Chem. 2015 (2015) Article ID 435129 (8 pages); https://doi.org/10.1155/2015/435129

63. G. L. Reid, J. Morgado, K. Barnett, B. Harrington, J. Wang, J. Harwood and D. Fortin, Analytical Quality by Design (AQbD) in pharmaceutical development, Am. Pharm. Rev., August 27, 2013; https://www.americanpharmaceuticalreview.com/Featured-Articles/144191-Analytical-Quality-byDesign-AQbD-in-Pharmaceutical-Development/

64. X. Yu, L. X. Yu, Y. Teng, D. K. Gaglani, B. D. Rege and S. Rosencrance, Implementation of Pharmaceutical Quality by Design in Wet Granulation, in Handbook of Pharmaceutical Wet Granulation - Theory and Practice in a Quality by Design Paradigm (Eds. A. S. Narang and S. I. F. Badawy), Elsevier, Amsterdam 2019, pp. 703-733.

65. L. Eriksson, E. Johansson, N. Kettaneh-Wold, C. Wikström and S. Wold, Design of Experiments, Principles and Applications, $3^{\text {rd }}$ Edition, Umetrics, Umeå 2000.

66. Z. I. Szabó, B. Székely-Szentmiklósi, B. Deák, I. Székely-Szentmiklósi, B. Kovács, K. Zöldi and E. Sipos, Study of the effect of formulation variables on the characteristics of combination tablets containing enalapril maleate and indapamide as active substances using experimental design, Acta Pharm. 66 (2016) 191-206; https://doi.org/10.1515/acph-2016-0019 
67. B. Rambali, L. Baert and D. L. Massart, Using experimental design to optimize the process parameters in fluidized bed granulation on a semi-full scale, Int. J. Pharm. 220 (2001) 149-160; https://doi. org/10.1016/S0378-5173(01)00658-5

68. J. Djuris, D. Medarevic, M. Krstic, Z. Djuric and S. Ibric, Application of quality by design concepts in the development of fluidized bed granulation and tableting processes, J. Pharm. Sci. 102 (2013) 1869-1882; https://doi.org/10.1002/jps.23530

69. I. Aleksić, J. Đuriš, I. Ilić, S. Ibrić, J. Parojčić and S. Srčič, In silico modeling of in situ fluidized bed melt granulation, Int. J. Pharm. 466 (2014) 21-30; https://doi.org/10.1016/j.ijpharm.2014.02.045

70. A. S. Zidan, M. Ebeed, H. Elghamry and A. Badawy, Nicotinamide pelletization by fluidized hot melt granulation: L18 Hunter design to screen high risk variables, Int. J. Pharm. 466 (2014) 83-95; https://doi.org/10.1016/j.ijpharm.2014.03.008

71. H. F. Santos Souza, D. Real, D. Leonardi, S. C. Rocha, V. Alonso, E. Serra, A. M. Silber and C. J. Salomon, Development and in vitro/in vivo evaluation of a novel benznidazole liquid dosage form using a quality-by-design approach, Trop. Med. Int. Heal. 22 (2017) 1514-1522; https://doi.org/10.1111/ tmi.12980

72. M. Cirri, F. Maestrelli, P. Mura, C. Ghelardini and L. Di Cesare Mannelli, Combined approach of cyclodextrin complexationand nanostructured lipid carriers for the development of a pediatric liquid oral dosage form of hydrochlorothiazide, Pharmaceutics 10 (2018) Article ID 287 (17 pages); https://doi.org/10.3390/pharmaceutics10040287

73. J. Joseph, B. N. V. Hari and D. R. Devi, Experimental optimization of lornoxicam liposomes for sustained topical delivery, Eur. J. Pharm. Sci. 112 (2018) 38-51; https://doi.org/10.1016/j.ejps.2017.10.032

74. B. Sylvester, A. Porfire, D. M. Muntean, L. Vlase, L. Lupuț, E. Licarete, A. Sesarman, M. C. Alupei, M. Banciu, M. Achim and I. Tomuță, Optimization of prednisolone-loaded long-circulating liposomes via application of Quality by Design (QbD) approach, J. Liposome Res. 28 (2018) 49-61; https:// doi.org/10.1080/08982104.2016.1254242

75. S. Alam, M. Aslam, A. Khan, S. S. Imam, M. Aqil, Y. Sultana and A. Ali, Nanostructured lipid carriers of pioglitazone for transdermal application: From experimental design to bioactivity detail, Drug Deliv. 23 (2016) 601-609; https://doi.org/10.3109/10717544.2014.923958

76. V. Sutariya, A. Groshev, P. Sadana, D. Bhatia and Y. Pathak, Artificial neural network in drug delivery and pharmaceutical research, Open Bioinform. J. 7 (2014) 49-62; https://doi.org/10.2174/ 1875036201307010049

77. M. Puri, A. Solanki, T. Padawer, S. M. Tipparaju, W. A. Moreno and Y. Pathak, Introduction to Artificial Neural Network (Ann) as a Predictive Tool for Drug Design, Discovery, Delivery, and Disposition: Basic Concepts and Modeling, in Artificial Neural Network for Drug Design, Delivery and Disposition (Eds. M. Puri, Y. Pathak, V. K. Sutariya, S. Tipparaju and W. Moreno), Elsevier Amsterdam 2016, pp. 3-13.

78. F. Amato, A. López, E. M. Peña-Méndez, P. Vaňhara, A. Hampl and J. Havel, Artificial neural networks in medical diagnosis, J. Appl. Biomed. 11 (2013) 47-58; https://doi.org/10.2478/v10136-012-0031-x

79. V. Mandlik, P. R. Bejugam and S. Singh, Application of artificial neural networks in modern drug discovery, in: Artificial. Neural Network for Drug Design, Delivery and Disposition (Eds. M. Puri, Y. Pathak, V. K. Sutariya, S. Tipparaju and W. Moreno), Elsevier, Amsterdam 2016, pp. 123-139.

80. P. S. Rajpal, K. S. Shishodia and G. S. Sekhon, An artificial neural network for modeling reliability, availability and maintainability of a repairable system, Reliab. Eng. System. Saf. 91 (2006) 809-819; https://doi.org/10.1016/j.ress.2005.08.004

81. M. Bianchini and F. Scarselli, On the complexity of neural network classifiers: A comparison between shallow and deep architectures, IEEE Trans. Neural Networks Learn. Syst. 25 (2014) 1553-1565; https://doi.org/10.1109/TNNLS.2013.2293637

82. D. A. Winkler and T. C. Le, Performance of deep and shallow neural networks, the universal approximation theorem, activity cliffs, and QSAR, Mol. Inform. 36 (2017) Article ID 1600118; https:// doi.org/10.1002/minf.201600118 
83. M. Z. Alom, T. M. Taha, C. Yakopcic, S. Westberg, P. Sidike, M. S. Nasrin, M. Hasan, B. C. Van Essen, A. A. S. Awwal and V. K. Asari, A state-of-the-art survey on deep learning theory and architectures, Electronics 8 (2019) Article ID 292; https://doi.org/10.3390/electronics8030292

84. N. K. Chauhan and K. Singh, A review on conventional machine learning vs deep learning, in: 2018 International Conference on Computing, Power and Communication Technologies (GUCON), Greater Noida (UP, India), Sept. 28-29, 2018; IEEE, pp. 347-352; https://doi.org/10.1109/GUCON.2018.8675097

85. T. I. Poznyak, I. Chairez Oria and A. S. Poznyak, Background on Dynamic Neural Networks, in Ozonation and Biodegradation in Environmental Engineering, Dynamic Neural Network Approach (Eds. T. I. Poznyak, I. Chairez and A. S. Poznyak), Elsevier, Amsterdam 2019, pp. 57-74.

86. N. Lanzetti, Y. Z. Lian, A. Cortinovis, L. Dominguez, M. Mercangöz and C. Jones, Recurrent neural network based MPC for process industries, 2019 18th European Control Conference (ECC), Naples (Italy), June 25-28, 2019, IEEE, pp. 1005-1010, https://doi.org/10.23919/ECC.2019.8795809

87. B. Zhang, X. Sun, S. Liu and X. Deng, Recurrent neural network-based model predictive control for multiple unmanned quadrotor formation flight, Int. J. Aerosp. Eng. 2019 (2019) (18 pages); https://doi. org/10.1155/2019/7272387

88. M. Ankith, S. S. Teja and N. Demodharan, Artifical Neural networks: functioning and applications in pharmaceutical industry, Int. J. Appl. Pharm. 10 (2018) 28-33; https://doi.org/10.22159/ ijap.2018v10i5.28300

89. M. Pishnamazi, H. Y. Ismail, S. Shirazian, J. Iqbal, G. M. Walker and M. N. Collins, Application of lignin in controlled release: development of predictive model based on artificial neural network for API release, Cellulose 26 (2019) 6165-6178; https://doi.org/10.1007/s10570-019-02522-w

90. C. Prithviraj, P. Versha, C. D. Debarupa and G. Amitava, Application of artificial neural network model in predicting physicochemical characteristics of pharmaceutically developed wafers of loratadine, Asian J. Pharm. 9 (2015) 44-48; https://doi.org/10.4103/0973-8398.150036

91. B. Aksu, G. Yegen, S. Purisa, E. Cevher and Y. Ozsoy, Optimisation of ondansetron orally disintegrating tablets using artificial neural networks, Trop. J. Pharm. Res. 13 (2014) 1374-1383; https://doi. org/10.4314/tjpr.v13i9.1

92. R. Han, Y. Yang, X. Li and D. Ouyang, Predicting oral disintegrating tablet formulations by neural network techniques, Asian J. Pharm. Sci. 13 (2018) 336-342; https://doi.org/10.1016/j.ajps.2018.01.003

93. M. Ilić, J. Đuriš, I. Kovačević, S. Ibrić and J. Parojčić, In vitro - in silico - in vivo drug absorption model development based on mechanistic gastrointestinal simulation and artificial neural networks: Nifedipine osmotic release tablets case study, Eur. J. Pharm. Sci. 62 (2014) 212-218; https://doi. org/10.1016/j.ejps.2014.05.030

94. D. L. Galata, A. Farkas, Z. Könyves, L. A. Mészáros, E. Szabó, I. Csontos, A. Pálos, G. Marosi, Z. K. Nagy and B. Nagy, Fast, spectroscopy-based prediction of in vitro dissolution profile of extended release tablets using artificial neural networks, Pharmaceutics 11 (2019) Article ID 400 (18 pages); https://doi.org/10.3390/pharmaceutics11080400

95. B. Nagy, D. Petra, D. L. Galata, B. Démuth, E. Borbás, G. Marosi, Z. K. Nagy and A. Farkas, Application of artificial neural networks for process analytical technology-based dissolution testing, Int. J. Pharm. 567 (2019) Article ID 118464; https://doi.org/10.1016/j.ijpharm.2019.118464

96. M. Zandkarimi, M. Shafiei, F. Hadizadeh, M. Ali Darbandi and K. Tabrizian, Prediction of pharmacokinetic parameters using a genetic algorithm combined with an artificial neural network for a series of alkaloid drugs, Sci. Pharm. 82 (2014) 53-70; https://doi.org/10.3797/scipharm.1306-10

97. W. C. Wong, E. Chee, J. Li and X. Wang, Recurrent neural network-based model predictive control for continuous pharmaceutical manufacturing, Mathematics 6 (2018) Article ID 242; https://doi. org/10.3390/math6110242

98. P. Oliveri and M. Forina, Data Analysis and Chemometrics, in Chemical Analysis of Food: Techniques and Applications (Ed. Y. Pico), Elsevier, Amsterdam 2012, pp. 25-57. 
99. S. Grimnes and O. G. Martinsen, Data and Models, in Bioimpedance and Bioelectricity Basics (Eds. S. Grimnes and O. G. Martinsen), $3^{\text {rd }}$ ed, Elsevier, Amsterdam 2015, pp. 329-404.

100. J. S. Markowitz, Multivariate Analysis, in Mortality and Its Risk Factors Among Profession Athletes (Ed. J. S. Markowitz), Springer Nature, Cham (Switzerland) 2018, pp. 71-81.

101. A. P. Ferreira and M. Tobyn, Multivariate analysis in the pharmaceutical industry: enabling process understanding and improvement in the PAT and QbD era, Pharm. Dev. Technol. 20 (2015) 513-527; https://doi.org/10.3109/10837450.2014.898656

102. I. Singh, P. Juneja, B. Kaur and P. Kumar, Pharmaceutical applications of chemometric techniques, ISRN Anal. Chem. 2013 (2013) Article ID 795178 (13 pages); https://doi.org/10.1155/2013/795178

103. I. T. Jolliffe and J. Cadima, Principal component analysis: A review and recent developments, Philos. Trans. R. Soc. A 374 (2016) Article ID 20150202 (16 pages); https://doi.org/10.1098/rsta.2015.0202

104. S. Maitra and J. Yan, Principle Component Analysis and Partial Least Squares: Two dimension Reduction Techniques for Regression, in Applying Multivariate Statistical Models, 2008 Discussion Paper Program, June 15-18, 2008, Casualty Actuarial Society, Québec City, pp.79-90.

105. R. Roopwani, Z. Shi and I. S. Buckner, Application of principal component analysis (PCA) to evaluating the deformation behaviors of pharmaceutical powders, J. Pharm. Innov. 8 (2013) 121-130; https://doi.org/10.1007/s12247-013-9153-2

106. S. Bhattacharya, S. Mishra and B. G. Prajapati, Design and development of docetaxel solid selfmicroemulsifying drug delivery system using principal component analysis and D-optimal design, Asian J. Pharm. 12 (2018) S122-S144.

107. N. L. Calvo, T. S. Kaufman and R. M. Maggio, Mebendazole crystal forms in tablet formulations. An ATR-FTIR/chemometrics approach to polymorph assignment, J. Pharm. Biomed. Anal. 122 (2016) 157-165; https://doi.org/10.1016/j.jpba.2016.01.035

108. S. Glavanović, M. Glavanović and V. Tomišić, Simultaneous quantitative determination of paracetamol and tramadol in tablet formulation using UV spectrophotometry and chemometric methods, Spectrochim. Acta A 157 (2016) 258-264; https://doi.org/10.1016/j.saa.2015.12.020

109. A. Mostafa, A. El Gindy and S. Emara, Simultaneous spectrophotometric estimation of bisoprolol fumarate and hydrochlorothiazide in tablet formulation using partial least-squares, principal component regression multivariate calibrations and RP-HPLC methods, J. Anal. Pharm. Res. 4 (2017) Article ID 00124 (9 pages); https://doi.org/10.15406/japlr.2017.04.00124

110. B. M. Marson, R. de Oliveira Vilhena, C. R. de Souza Madeira, F. L. D. Pontes, M. S. Piantavini and R. Pontarolo, Simultaneous quantification of artesunate and mefloquine in fixed-dose combination tablets by multivariate calibration with middle infrared spectroscopy and partial least squares regression, Malaria J. 15 (2016) Article ID 109; https://doi.org/10.1186/s12936-016-1157-1

111. J. Silva, M. Mendes, T. Cova, J. Sousa, A. Pais and C. Vitorino, Unstructured formulation data analysis for the optimization of lipid nanoparticle drug delivery vehicles, AAPS PharmSciTech 19 (2018) 2383-2394; https://doi.org/10.1208/s12249-018-1078-0

112. S. F. B. Ali, Z. Rahman, S. Dharani, H. Afrooz and M. A. Khan, Chemometric models for quantification of carbamazepine anhydrous and dihydrate forms in the formulation, J. Pharm. Sci. 108 (2019) 1211-1219; https://doi.org/10.1016/j.xphs.2018.10.023

113. V. Arabzadeh, M. R. Sohrabi, N. Goudarzi and M. Davallo, Using artificial neural network and multivariate calibration methods for simultaneous spectrophotometric analysis of emtricitabine and tenofovir alafenamide fumarate in pharmaceutical formulation of HIV drug, Spectrochim. Acta A 215 (2019) 266-275; https://doi.org/10.1016/j.saa.2019.02.077

114. G. Ioele, M. de Luca, E. Dinç, F. Oliverio and G. Ragno, Artificial neural network combined with principal component analysis for resolution of complex pharmaceutical formulations, Chem. Pharm. Bull. 59 (2011) 35-40; https://doi.org/10.1248/cpb.59.35 
115. R. W. Bondi and J. K. Drennen, Quality by Design and the Importance of PAT in QbD, in Separation Science and Technology (Eds. S. Ahuja and S. Scypinski), Elsevier, Amsterdam 2020, Vol. 10, pp. 195-224.

116. S. Laske, A. Paudel, O. Scheibelhofer, S. Sacher, T. Hoermann, J. Khinast, A. Kelly, J. Rantannen, O. Korhonen, F. Stauffer, F. De Leersnyder, T. De Beer, J. Mantanus, P. F. Chavez, B. Thoorens, P. Ghiotti, M. Schubert, P. Tajarobi, G. Haeffler, S. Lakio, M. Fransson, A. Sparen, S. Abrahmsen-Alami, S. Folestad, A. Funke, I. Backx, B. Kavsek, F. Kjell, M. Michaelis, T. Page, J. Palmer, A. Schaepman, S. Sekulic, S. Hammond, B. Braun and B. Colegrove, A review of PAT strategies in secondary solid oral dosage manufacturing of small molecules, J. Pharm. Sci. 106 (2017) 667-672; https://doi. org/10.1016/j.xphs.2016.11.011

117. T. De Beer, A. Burggraeve, M. Fonteyne, L. Saerens, J. P. Remon and C. Vervaet, Near infrared and Raman spectroscopy for the in-process monitoring of pharmaceutical production processes, Int. J. Pharm. 417 (2011) 32-47; https://doi.org/10.1016/j.ijpharm.2010.12.012

118. T. Helešicová, T. Pekárek and P. Matějka, The influence of different acquisition settings and the focus adjustment on Raman spectral maps of pharmaceutical tablets, J. Drug Delivery Sci. Tehnol. 47 (2018) 386-394; https://doi.org/10.1016/j.jddst.2018.08.002

119. H. Mitsutake, S. R. Castro, E. de Paula, R. J. Poppi, D. N. Rutledge and M. C. Breitkreitz, Comparison of different chemometric methods to extract chemical and physical information from Raman images of homogeneous and heterogeneous semi-solid pharmaceutical formulations, Int. J. Pharm. 552 (2018) 119-129; https://doi.org/10.1016/j.ijpharm.2018.09.058

120. K. Nomura, V. Titapiwatanakun, H. Hisada, T. Koide and T. Fukami, In situ monitoring of the crystalline state of active pharmaceutical ingredients during high-shear wet granulation using a low-frequency Raman probe, Eur. J. Pharm. Biopharm. 147 (2020) 1-9; https://doi.org/10.1016/j. ejpb.2019.12.004

121. A. Gavan, S. Iurian, T. Casian, A. Porfire, S. Porav, I. Voina, A. Oprea and I. Tomuta, Fluidised bed granulation of two APIs: QbD approach and development of a NIR in-line monitoring method, Asian J. Pharm. Sci. 15 (2020) 506-517; https://doi.org/10.1016/j.ajps.2019.03.003

122. T. Casian, A. Reznek, A. L. Vonica-Gligor, J. Van Renterghem, T. De Beer and I. Tomuță, Development, validation and comparison of near infrared and Raman spectroscopic methods for fast characterization of tablets with amlodipine and valsartan, Talanta 167 (2017) 333-343; https://doi. org/10.1016/j.talanta.2017.01.092

123. H. Wu and M. Khan, THz spectroscopy: An emerging technology for pharmaceutical development and pharmaceutical Process analytical technology (PAT) applications, J. Mol. Struct. 1020 (2012) 112-120; https://doi.org/10.1016/j.molstruc.2012.04.019

124. P. F. Taday, D. Van Der Weide, K. Wood, M. Chamberlain, H. Roskos, C. Phillips, D. Newnham, M. Towrie and I. Appelquist, Applications of terahertz spectroscopy to pharmaceutical sciences, Philos. Trans. R. Soc. A 362 (2004) 351-364; https://doi.org/10.1098/rsta.2003.1321

125. Y. B. Monakhova, U. Holzgrabe and B. W. K. Diehl, Current role and future perspectives of multivariate (chemometric) methods in NMR spectroscopic analysis of pharmaceutical products, J. Pharm. Biomed. Anal. 147 (2018) 580-589; https://doi.org/10.1016/j.jpba.2017.05.034

126. K. Korasa and F. Vrečer, Overview of PAT process analysers applicable in monitoring of film coating unit operations for manufacturing of solid oral dosage forms, Eur. J. Pharm. Sci. 111 (2018) 278-292; https://doi.org/10.1016/j.ejps.2017.10.010

127. E. M. Hansuld and L. Briens, A review of monitoring methods for pharmaceutical wet granulation, Int. J. Pharm. 472 (2014) 192-201; https://doi.org/10.1016/j.ijpharm.2014.06.027

128. X. Hu, J. C. Cunningham and D. Winstead, Study growth kinetics in fluidized bed granulation with at-line FBRM, Int. J. Pharm. 347 (2008) 54-61; https://doi.org/10.1016/j.ijpharm.2007.06.043 
129. F. Alshihabi, T. Vandamme and G. Betz, Focused beam reflectance method as an innovative (PAT) tool to monitor in-line granulation process in fluidized bed, Pharm. Dev. Technol. 18 (2013) 73-84; https://doi.org/10.3109/10837450.2011.627868

130. A. S. Narang, T. Stevens, K. Macias, S. Paruchuri, Z. Gao and S. Badawy, Application of in-line focused beam reflectance measurement to brivanib alaninate wet granulation process to enable scale-up and attribute-based monitoring and control strategies, J. Pharm. Sci. 106 (2017) 224-233; https://doi.org/10.1016/j.xphs.2016.08.025

131. U. Verma, J. B. Naik, J. S. Patil and S. K. Yadava, Screening of process variables to enhance the solubility of famotidine with 2-hydroxypropyl- $\beta$-cyclodextrin \& PVP K-30 by using Plackett-Burman design approach, Mater. Sci. Eng. C 77 (2017) 282-292; https://doi.org/10.1016/j.msec.2017.03.238

132. A. Anand, G. Singh and S. A. Saraf, Plackett-Burman design as a tool for screening and process optimization of rivastigmine-loaded lipid nanocarriers, Asian J. Pharm. Clin. Res. 11 (2018) 155-158; https://doi.org/10.22159/ajpcr.2018.v11i12.28066

133. K. M. Hosny, O. A. A. Ahmed, U. A. Fahmy and H. M. Alkhalidi, Nanovesicular systems loaded with a recently approved second generation type- 5 phospodiesterase inhibitor (avanafil): I. Plackett-Burman screening and characterization, J. Drug Deliv. Sci. Technol. 43 (2018) 154-159; https://doi. org/10.1016/j.jddst.2017.10.009

134. T. Alam, S. Khan, B. Gaba, M. F. Haider, S. Baboota and J. Ali, Adaptation of Quality by Designbased development of isradipine nanostructured-lipid carrier and its evaluation for in vitro gut permeation and in vivo solubilization fate, J. Pharm. Sci. 107 (2018) 2914-2926; https://doi.org/10.1016/j. xphs.2018.07.021

135. S. S. Chudiwal and M. H. G. Dehghan, Quality by design (QbD) approach for design and development of drug-device combination products: a case study on flunisolide nasal spray, Pharm. Dev. Technol. 23 (2018) 1077-1087; https://doi.org/10.1080/10837450.2016.1236130

136. N. Jaipakdee, E. Limpongsa and T. Pongjanyakul, Optimization of minoxidil microemulsions using fractional factorial design approach, Pharm. Dev. Technol. 21 (2016) 86-97; https://doi.org/10.310 9/10837450.2014.971375

137. T. Adebileje, S. Adebileje and P. O. Aye, Ciprofloxacin hydrochloride encapsulated into PLGA nanoparticles for drug delivery application: Fractional factorial design, Open Access Library J. 5 (2018) e4294; https://doi.org/10.4236/oalib.1104294

138. A. Jain, T. Sharma, G. Sharma, R. K. Khurana, O. P. Katare and B. Singh, QbD-driven analytical method development and validation for raloxifene hydrochloride in pure drug and solid oral dosage form, Anal. Chem. Lett. 9 (2019) 463-477; https://doi.org/10.1080/22297928.2019.1624193

139. M. J. Ramalho, J. A. Loureiro, M. A. N. Coelho and M. C. Pereira, Factorial design as a tool for the optimization of PLGA nanoparticles for the co-delivery of temozolomide and O6-benzylguanine, Pharmaceutics 11 (2019) Article ID 401; https://doi.org/10.3390/pharmaceutics11080401

140. A. Kumar, A. Nayak and S. Ghatuary, Design, optimization and characterization of a transferosomal gel of acyclovir for effective treatment of Herpes zoster, J. Drug Deliv. Ther. 9 (2019) 712-721; https://doi.org/10.22270/jddt.v9i4-A.3556

141. C. Roy and J. Chakrabarty, Quality by design-based development of a stability-indicating RPHPLC method for the simultaneous determination of methylparaben, propylparaben, diethylamino hydroxybenzoyl hexyl benzoate, and octinoxate in topical pharmaceutical formulation, Sci. Pharm. 82 (2014) 519-539; https://doi.org/10.3797/scipharm.1312-20

142. A. R. Fernandes, N. R. Ferreira, J. F. Fangueiro, A. C. Santos, F. J. Veiga, C. Cabral, A. M. Silva and E. B. Souto, Ibuprofen nanocrystals developed by 22 factorial design experiment: A new approach for poorly water-soluble drugs, Saudi Pharm. J. 25 (2017) 1117-1124; https://doi.org/10.1016/j. jsps.2017.07.004 
143. H. Patel, H. Patel, M. Gohel and S. Tiwari, Dissolution rate improvement of telmisartan through modified MCC pellets using 32 full factorial design, Saudi Pharm. J. 24 (2016) 579-587; https://doi. org/10.1016/j.jsps.2015.03.007

144. U. Verma, R. Rajput and J. B. Naik, Development and characterization of fast dissolving film of chitosan embedded famotidine using $3^{2}$ full factorial design approach, Mater. Today Proc. 5 (2018) 408-414; https://doi.org/10.1016/j.matpr.2017.11.099

145. S. M. Soliman, N. S. Abdelmalak, O. N. El-Gazayerly and N. Abdelaziz, Novel non-ionic surfactant proniosomes for transdermal delivery of lacidipine: optimization using $2^{3}$ factorial design and in vivo evaluation in rabbits, Drug Deliv. 23 (2016) 1608-1622; https://doi.org/10.3109/10717544.2015.11 32797

146. R. R. Pereira, M. Testi, F. Rossi, J. O. C. Silva Junior, R. M. Ribeiro-Costa, R. Bettini, P. Santi, C. Padula and F. Sonvico, Ucuùba (Virola surinamensis) fat-based nanostructured lipid carriers for nail drug delivery of ketoconazole: Development and optimization using Box-Behnken design, Pharmaceutics 11 (2019) Article ID 284; https://doi.org/10.3390/pharmaceutics11060284

147. P. Kraisit and N. Sarisuta, Development of triamcinolone acetonide-loaded nanostructured lipid carriers (NLCs) for buccal drug delivery using the Box-Behnken design, Molecules 23 (2018) Article ID 982; https://doi.org/10.3390/molecules23040982

148. V. Londhe and R. Shirsat, Formulation and characterization of fast-dissolving sublingual film of iloperidone using Box-Behnken design for enhancement of oral bioavailability, AAPS PharmSciTech 19 (2018) 1392-1400; https://doi.org/10.1208/s12249-018-0954-y

149. K. Ghosal, D. Ghosh and S. K. Das, Preparation and evaluation of naringin-loaded polycaprolactone microspheres based oral suspension using Box-Behnken design, J. Mol. Liq. 256 (2018) 49-57; https://doi.org/10.1016/j.molliq.2018.02.024

150. M. Gad, H. E Zaazaa, S. M. Amer and M. A. Korany, Quality by Design approach for establishment of stability indicating method for determination of cefditoren pivoxil, J. Pharm. Anal. Insights 2 (2017) (7 pages); https://doi.org/10.16966/2471-8122.112

151. T. E. Yalcin, S. Ilbasmis-Tamer and S. Takka, Development and characterization of gemcitabine hydrochloride loaded lipid polymer hybrid nanoparticles (LPHNs) using central composite design, Int. J. Pharm. 548 (2018) 255-262; https://doi.org/10.1016/j.ijpharm.2018.06.063

152. U. C. Oz, B. Küçüktürkmen, B. Devrim, O. M. Saka and A. Bozkir, Development and optimization of alendronate sodium loaded PLGA nanoparticles by central composite design, Macromol. Res. 27 (2019) 857-866; https://doi.org/10.1007/s13233-019-7119-z

153. N. Sharma and S. Singh, Central composite designed ezetimibe solid dispersion for dissolution enhancement: Synthesis and in vitro evaluation, Ther. Deliv. 10 (2019) 643-658; https://doi. org/10.4155/tde-2019-0063

154. A. R. Fares, A. N. Elmeshad and M. A. A. Kassem, Enhancement of dissolution and oral bioavailability of lacidipine via pluronic P123/F127 mixed polymeric micelles: Formulation, optimization using central composite design and in vivo bioavailability study, Drug Deliv. 25 (2018) 132-142; https://doi.org/10.1080/10717544.2017.1419512

155. S. Jebali, C. Belgacem, M. R. Louhaichi, S. Bahri and L. L. El Atarche, Application of factorial and Doehlert designs for the optimization of the simultaneous separation and determination of antimigraine drugs in pharmaceutical formulations by RP-HPLC-UV, Int. J. Anal. Chem. 2019 (2019) Article ID 9685750 (11 pages); https://doi.org/10.1155/2019/9685750 